\title{
EUV spectral energy distribution of quasars restored from associated absorbers ${ }^{\star}$
}

\author{
S. A. Levshakov ${ }^{1,3, \star \star}$, I. I. Agafonova ${ }^{2,3}$, D. Reimers ${ }^{1}$, J. L. Hou ${ }^{3}$, and P. Molaro ${ }^{4}$ \\ 1 Hamburger Sternwarte, Universität Hamburg, Gojenbergsweg 112, 21029 Hamburg, Germany \\ e-mail: lev@astro.ioffe.rssi.ru \\ 2 Ioffe Physico-Technical Institute, Polytekhnicheskaya Str. 26, 194021 St. Petersburg, Russia \\ 3 Shanghai Astronomical Observatory, 80 Nandan Road, Shanghai 200030, PR China \\ 4 Osservatorio Astronomico di Trieste, via G. B. Tiepolo 11, 34131 Trieste, Italy
}

Received 20 November 2007 / Accepted 5 February 2008

\section{ABSTRACT}

\begin{abstract}
Aims. We reconstruct the spectral shape of the quasar ionizing radiation in the extreme-UV range (1 Ryd $\leq E<10$ Ryd) from the analysis of narrow absorption lines (NAL) of the associated systems.

Methods. The computational technique for inverse spectroscopic problems - a Monte Carlo Inversion augmented by the spectral shape recovering procedure and modified to account for the incomplete coverage of the light source is used to analyze observations. Results. The ionizing spectra responsible for the ionization structure of the NAL systems require an intensity depression at $E>4$ Ryd which is attributed to the He II Lyman continuum opacity $\left(\tau_{c}^{\text {He II }} \sim 1\right)$. A most likely source of this opacity is a quasar accretion disk wind. The corresponding column density of $\mathrm{HI}_{\mathrm{I}}$ in the wind is estimated as a few times $10^{16} \mathrm{~cm}^{-2}$. This amount of neutral hydrogen should cause a weak continuum depression at $\lambda \lesssim 912 \AA$ (rest-frame), and a broad and shallow absorption in HI Ly- $\alpha$. If the metallicity of the wind is high enough, other resonance lines of O VI, Ne VI-Ne VIII, etc. are expected. In the analyzed QSO spectra we do observe broad (stretching over 1000s km s${ }^{-1}$ ) and shallow $(\tau \ll 1)$ absorption troughs of H I Ly- $\alpha$ and O VI $\lambda \lambda 1031,1037$ as well as continuum depressions at $\lambda \lesssim 912 \AA$ which correspond to $N(\mathrm{HI}) \sim 5 \times 10^{16} \mathrm{~cm}^{-2}$. Observational data available in both the UV and X-ray ranges suggest that at least $\sim 50 \%$ of the quasar radiation passes through the gas opaque in the He II Lyman continuum. This means that the outcoming ionizing spectrum should have a pronounced intensity break at $E>4$ Ryd with the depth of this break depending on the angle with the rotational axis of the accretion disk (the larger the angle the deeper the break). The QSO spectra with a discontinuity at 4 Ryd can influence the rate of He II reionization in the intergalactic medium and partly explain the inhomogeneous (patchy) ionization structure of the intergalactic He II observed at $z \sim 3$.
\end{abstract}

Key words. cosmology: observations - line: formation - line: profiles - quasars: absorption lines

\section{Introduction}

The spectral energy distribution (SED) of the quasar/AGN outcoming radiation in the extreme-UV range (EUV, $\lambda<912 \AA$ ) is important for the interpretation of both emission and absorption spectra since this range contains the ionization thresholds of the commonly observed ions C II-C IV, N II-N V, O I-O VI, Si II-Si IV. The SED is accessible for direct measurements in the FUV-EUV ranges (rest-frame) for wavelengths $\lambda>300 \AA$ (e.g., Zheng et al. 1997; Telfer et al. 2002; Scott et al. 2004) and at shorter wavelengths only in the X-ray range for $\lambda<40 \AA$ (e.g., Piconcelli et al. 2005; Brocksopp et al. 2006; Costantini et al. 2007). In the intermediate range ( $40 \AA<\lambda<300 \AA$ ), direct measurements of the outcoming radiation of QSOs with redshifts $z<2$ are prevented by the Galactic absorption, and for QSOs with $z>2$ are, in principle, impossible due to the intergalactic gas opacity in He II Ly- $\alpha$.

The gap in the SED between $\lambda=912 \AA$ and soft X-ray range usually is approximated by a simple power law, $F_{v} \propto$ $v^{-\alpha}$. However, this part of the QSO continuum radiation is

\footnotetext{
^ Based on observations obtained at the VLT Kueyen telescope (ESO, Paranal, Chile), and at the HST.

$\star \star$ On leave from the Ioffe Physico-Technical Institute, St. Petersburg, Russia.
}

responsible for the ionization state of the so-called narrow absorption line (NAL) systems - metal systems arising in the gas located close to the quasar host galaxy - and can be reconstructed from their analysis.

The computational procedure aimed at restoring the spectral shape of the underlying ionizing radiation from ions observed in optically thin metal absorption-line systems is described in Agafonova et al. (2005, 2007). Applied to the analysis of NAL systems identified in the spectrum of the quasar HE 0141-3932 with $z_{\mathrm{em}}=1.8$ (Reimers et al. 2005), this procedure resulted in a SED that could not be described with a single power law index $\alpha$ : the EUV spectrum shows a step-like structure with a sharp intensity break at $\lambda \simeq 240 \AA$ ( $E \simeq 3.8 \mathrm{Ryd}$, shifted by $\sim 12 \AA$, or $\sim 16000 \mathrm{~km} \mathrm{~s}^{-1}$ redward from the He II Ly$\alpha$ edge $228 \AA$, or 4 Ryd) and a much slower intensity decay in the range 4 Ryd $<E<10$ Ryd. Being attributed to the Lyman continuum absorption in He II, the depth of the break gives the He II column density $N($ He II $)=0.7 \times 10^{18} \mathrm{~cm}^{-2}$. Similar spectra with breaks at $\sim 4$ Ryd corresponding to $N(\mathrm{He}$ II $)=(0.7-1.4) \times$ $10^{18} \mathrm{~cm}^{-2}$ were reconstructed from the intergalactic absorptionline systems with $z_{\mathrm{abs}} \lesssim 1.8$ (Fig. 20 and Table 4 in Agafonova et al. 2007). Since there are indications that a large part of the QSO/AGN emitted radiation at this redshift remains unprocessed by the IGM, we argued that these spectra may represent 
the energy distribution of the outcoming quasar radiation. In the present paper, we continue the study of the intrinsic quasar SED using four associated (i.e. physically related to QSO) NAL systems identified in spectra of high-redshift $\left(2<z_{\mathrm{em}}<3\right)$ QSOs.

Concerning the selection of the NAL systems considered in the paper the following is to be noted. The metal NAL systems are in general not rare - they are observed in 30\%-50\% of quasar spectra (Misawa et al. 2007). However, for most of them the observed wavelength coverage and the ionization conditions in the absorbing gas result in the fact that at best only three highly ionized doublets C IV $\lambda \lambda 1548,1551 \AA$, $\mathrm{N} v \lambda \lambda 1238,1242 \AA$, and O VI $\lambda \lambda 1031,1037 \AA$ are available for study. The column densities of these absorption lines can be reproduced by a wide variety of the ionizing spectra and, thus, do not allow us to distinguish between specific SEDs. Another aggravating factor while working with NAL systems is that the estimation of the ion column densities is often hampered by effects like partial coverage of the background source of the continuum radiation and/or line blending.

This explains the selection criteria that governed the search for the appropriate associated systems: (1) the presence of many lines of different ions - to obtain reasonable restrictions on the SED, (2) clear line profiles, and (3) the possibility of determining the covering factor with sufficiently high accuracy. These requirements significantly restricted the number of suitable systems, but the loss in the quantity was compensated for by the increased quality of the data, which allowed us to clarify some additional aspects of the physical properties of the NAL systems.

The paper is organized as follows. In Sect. 2 we reconstruct the SED of the intrinsic quasar EUV radiation from the analysis of quasar-related metal absorption-line systems identified in spectra of four QSOs with redshifts $z_{\mathrm{em}}=2.2-2.9$. The results obtained are discussed in Sect. 3, and summarized in Sect. 4.

\section{Analysis of individual NAL systems}

Absorption systems are analyzed by means of the Monte Carlo Inversion (MCI) procedure (Levshakov et al. 2000, 2003; Agafonova et al. 2005, 2007) which is based on the assumption that all lines observed in the metal system are formed in the same gas with fluctuating density and velocity fields. The procedure's inputs are the observed line profiles and the ionization curves for each ion (hydrogen + metals) included in the analysis, the outputs are parameters of the gas density and velocity distributions and individual metal abundances which are supposed to be constant within the absorber. The column densities of hydrogen and all metal ions are calculated as well. The ionization curves are computed with the photoionization code CLOUDY version 07.02.01 (last described by Ferland et al. 1998) which in turn uses as an input the adopted (trial) SED.

For the present work the computational procedure was modified as to account for the incomplete coverage of the background light source. With $C$ standing for the covering factor (fraction of the background source of the continuum radiation occulted by the absorbing cloud) and based on principles of geometrical optics one can easily determine the observed normalized intensity $I_{\lambda}$ within the absorption line profile as

$I_{\lambda}=C \exp \left(-\tau_{\lambda}\right)+(1-C)$.

The apparent optical depth $\tau_{\lambda}$ is calculated with Eq. (4) in Levshakov et al. (2000). The covering factor is assumed to be the same for the whole cloud, i.e. it does not depend on $\lambda$ (or, equivalently, on the radial velocity $v$ ). However, different covering factors are allowed for different ions.
It is well known that NAL systems with incomplete coverage often show $\lambda$-dependent covering factors which may be caused by the overlapping of clouds with different covering factors or by the interaction of several physical processes. In these cases the estimation of accurate column densities becomes problematic and in the present study such systems are not used for SED recovery.

The SED of the radiation that ionizes the NAL system is reconstructed according to the iterative procedure described in Agafonova et al. (2005, 2007). The procedure includes (1) the parameterization of the spectral shape by means of a set of variables (called "factors"), (2) the choice of a quantitative measure (called a "response") to evaluate the goodness of a trial spectral shape, and (3) the estimation of a direction in the factor space which leads to a spectrum with the better goodness. Moving along this direction, we come from the initial spectral shape to one with better characteristics concerning the fitting of the observed line intensities. Now the MCI calculations are carried out with this newly obtained ionizing spectrum and the whole procedure is repeated until the optimal spectral shape is found, i.e. one which allows us to reproduce the observed intensities of all lines without any physical inconsistencies.

All calculations throughout the paper were performed with laboratory wavelength and oscillator strengths taken from Morton (2003) for $\lambda>912 \AA$ and from Verner et al. (1994) for $\lambda<912 \AA$. Solar abundances were taken from Asplund et al. (2004). Note that their solar abundances of neon, $\mathrm{Ne} / \mathrm{H}=7.5 \times$ $10^{-5}$, and nitrogen, $\mathrm{N} / \mathrm{H}=6 \times 10^{-5}$, are 1.4 times $(0.15 \mathrm{dex})$ lower than those from Holweger (2001) which are used as default inputs in CLOUDY.

\subsection{NAL systems towards J 2233-606}

The quasar J 2233-606 $\left(z_{\mathrm{em}}=2.238\right)$ was observed both at the VLT/UVES and HST/STIS with a wide wavelength coverage from $2300 \AA$ to $\simeq 10000 \AA$ (Cristiani \& D'Odorico 2000). The spectrum reveals an associated complex consisting of several absorption systems extending over $1000 \mathrm{~km} \mathrm{~s}^{-1}$. Due to the wavelength coverage it becomes possible to identify not only the usually observed lines of the doublets CIV $\lambda \lambda 1548,1551$, $\mathrm{N} v \lambda \lambda 1238,1242$, and O VI $\lambda \lambda 1032,1037$, but also the lines of Ne VIII $\lambda \lambda 770,780$ (albeit in a very noisy part of the STIS spectrum).

The associated systems towards J 2233-606 have been studied twice - by Petitjean \& Srianand (1999) and by Gabel et al. (2006). Both groups determined the column densities of different species, $N_{i}$, using velocity-dependent covering factors and after that compared $N_{i}$ with the values predicted by models where simple power law SEDs in the range from UV to X-ray regions were assumed. It was concluded that such models cannot account for the observed strong Ne VIII absorption and that an additional high-ionization absorber is needed to reproduce the $\mathrm{Ne}$ VIII line. In the present section we re-analyze two absorption systems from this complex using a physical model of the associated absorber as outlined above. Namely, gas in the absorbing cloud has varying density and velocity and the covering factor is assumed to be identical for all points in the line profile (no velocity dependence). However, the covering factor can differ for individual ions since different ions trace different parts of the gas resulting in different solid angles subtended. 


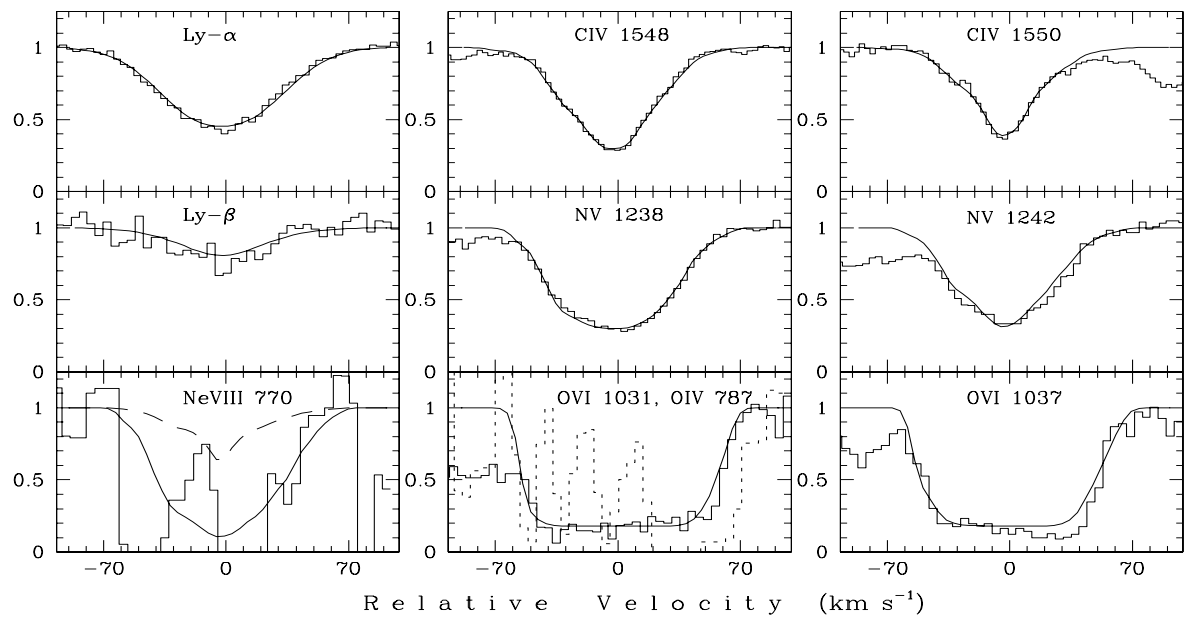

Fig. 1. Hydrogen and metal absorption lines from the $z_{\text {abs }}=2.198$ system towards J 2233-606 (solid-line histograms). The vertical axis is normalized intensity. The zero radial velocity is fixed at $z=2.1982$. Synthetic profiles are plotted with smooth curves. In panel with Ne VIII, the dashed curve corresponds to the profile predicted for the power law ionizing spectrum $F_{v} \propto v^{-1.5}$, and the smooth curve to the profile predicted for the SED with a break at $\sim 4$ Ryd (Fig. 3). The observed profile of O IV $\lambda 787 \AA$ (dotted-line histogram) overplotted on O VI $\lambda 1031$ is blended with Ly- $\gamma$ from the $z_{\mathrm{abs}}=1.59$ system in the range $\sim 20-70 \mathrm{~km} \mathrm{~s}^{-1}$.

\subsubsection{System at $z_{\mathrm{abs}}=2.198$}

Apart from metal doublets, this system also exhibits clear lines of hydrogen Ly- $\alpha$ and Ly- $\beta$ (Fig. 1). There are continuum windows in the QSO spectrum at the expected positions of C III $\lambda 977 \AA$ and SiIV $\lambda 1393 \AA$, and the expected position of O IV $\lambda 787 \AA$ is blended with Ly- $\gamma$ from the $z_{\mathrm{abs}}=1.59$ system (the O IV profile is overplotted on the panel with O VI $\lambda 1031 \AA$ in Fig. 1).

The first trial calculations were performed with the power law SED, $\alpha=1.5$. The objective function included hydrogen lines plus all lines of metal doublets mentioned above except for $\mathrm{Ne}$ VIII because of its too low $\mathrm{S} / \mathrm{N}$ ratio. Since the system under consideration does not show ionic lines of subsequent ionization stages, additional assumptions are required to fix the mean ionization parameter $U$. For the oversolar metallicity which is reliably reproduced for this system independent of the model assumptions, the usual constraint is the solar relative abundance of oxygen to carbon, i.e. $[\mathrm{C} / \mathrm{O}] \sim 0$.

The observed profiles of all doublets can be well described by a model with a single covering factor which takes individual values for each ion (smooth curves in Fig. 1). The estimated covering factors along with the column densities of ions are given in Table 1.

In the present case both the covering factors and the column densities are invariant and depend neither on the assumed SED nor on the calculation method. For instance, a commonly used deconvolution of the line profiles into subcomponents delivers the same values for the covering factors and column densities as obtained with the MCI procedure.

For the adopted SED $\left(v^{-1.5}\right)$ the ionization curves for ions C IV, OVI and Ne VIII are plotted with dotted lines in Fig. 2 with the shadowed area indicating the range of the ionization parameter $U$ inside the absorber. Both neon and oxygen are $\alpha$-elements and are expected to have the relative abundances $[\mathrm{Ne} / \mathrm{O}] \sim 0$. This gives a predicted column density $N_{\mathrm{Ne} \mathrm{VIII}} \sim 5 \times$ $10^{13} \mathrm{~cm}^{-2}$ with the corresponding synthetic profile shown by the dashed curve in the Ne VIII panel in Fig. 1. Obviously the line is too shallow to account for the observed Ne VIII absorption. This is valid for all power law SEDs since the arrangement of C IV, O VI and Ne VIII ionization curves does not vary with
Table 1. Parameters of the NAL system $z_{\text {abs }}=2.198$ towards J 2233606.

\begin{tabular}{ccc}
\hline \hline Ion & $N_{i}, \mathrm{~cm}^{-2}$ & $C_{i}$ \\
\hline H I & $(1.1 \pm 0.1) \mathrm{E} 14$ & $0.60 \pm 0.03$ \\
C IV & $(1.7 \pm 0.1) \mathrm{E} 14$ & $0.72 \pm 0.01$ \\
N V & $(5.3 \pm 0.8) \mathrm{E} 14$ & $0.70 \pm 0.02$ \\
O VI & $(3.4 \pm 0.7) \mathrm{E} 15$ & $0.82 \pm 0.02$ \\
\hline
\end{tabular}

increasing/decreasing spectral index: the curves are simply shifted along the $U$ axis to the right/left, but in the $U$ range that ensures the ratio $[\mathrm{C} / \mathrm{O}] \sim 0$ the fraction of Ne VIII remains too small.

The situation changes for a SED with a break around 4 Ryd like the one plotted with a solid line in Fig. 3. The ionization curves for this SED are given in the right hand part of Fig. 2 (solid lines) with the shadowed area indicating the range of the fractions of $\mathrm{C} I V$ and $\mathrm{O}$ VI ensuring $[\mathrm{C} / \mathrm{O}] \sim 0$. Now the fraction of Ne VIII is nearly equal to that of O VI leading to the column density of Ne VIII an order of magnitude higher than from the power law SED: $N_{\mathrm{Ne} \mathrm{VIII}} \sim 5 \times 10^{14} \mathrm{~cm}^{-2}$. The corresponding synthetic profile for Ne VIII is plotted in Fig. 1 for the covering factor $C_{\mathrm{Ne} \text { VIII }}=0.9$ (smooth line). A noisy HST/STIS spectrum at the positions of Ne VIII $\lambda \lambda 770,780 \AA$ prevents an accurate determination of both the covering factor and column density, but it is clearly seen that the adopted - broken power law - SED produces $N_{\mathrm{Ne} \text { VIII }}$ consistent with observations. This SED also gives the following abundances: $[\mathrm{C}, \mathrm{O}, \mathrm{Ne} / \mathrm{H}]=0.5,[\mathrm{~N} / \mathrm{H}]=0.9-1.0$, i.e. compared to the solar values nitrogen is $2.5-3$ times more abundant than other elements.

The absence of low-ionization lines and the unknown metallicity of the $z_{\mathrm{abs}}=2.198$ absorber does not allow us to recover all the details of the underlying SED of the ionizing radiation. Numerical simulations with different types of SED show that the simultaneous existence of significant amounts of C IV, O VI and Ne VIII can be provided only by a sharp break in the spectrum around the energy of 4 Ryd. However, other spectral shape features are determined with much less accuracy. For instance, the positions of the ionization curves shown in Fig. 2 are insensitive to the spectral index $\alpha$ in the energy range below the discontinuity point $A$ (shown in Fig. 3) at 1 Ryd $<E<4$ Ryd. 


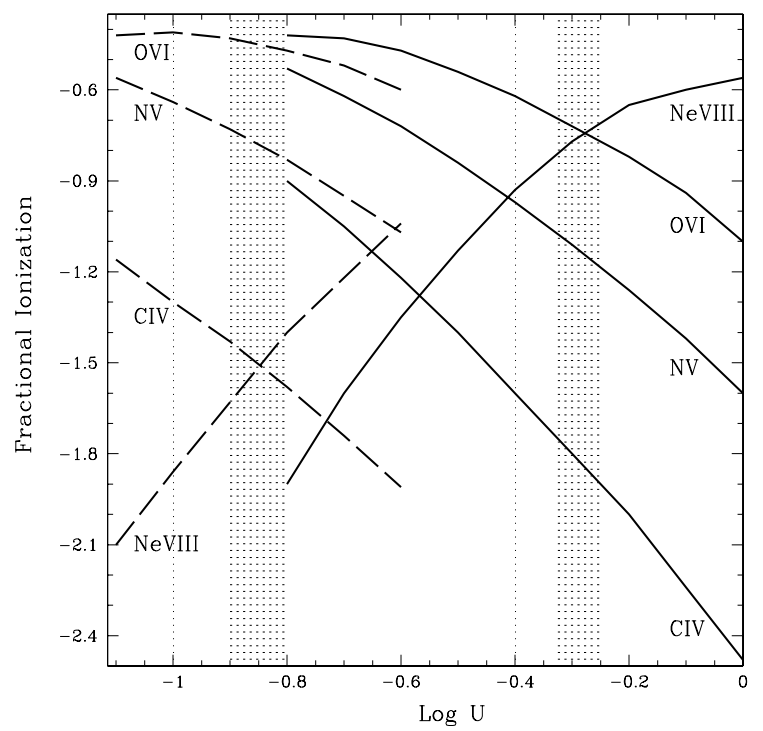

Fig. 2. Ionization fractions of different ions calculated for the power law ionizing spectrum $F_{v} \propto v^{-1.5}$ (dashed lines), and for the shown in Fig. 3 ionizing spectrum with a break at $\sim 4$ Ryd (solid lines). Calculations are performed with solar abundances and for the thermal and ionization equilibrium. See text for more details.

A deeper break after point $A$ does not affect the ionization curve of hydrogen, but shifts the ionization curves of other ions to the right. The condition $[\mathrm{C} / \mathrm{O}] \sim 0$ is now fulfilled at higher $U$ with a correspondingly lower fraction of neutral hydrogen thus, we obtain the same column density of Ne VIII but the metal content becomes lower: the dashed line SED in Fig. 3 gives a metallicity $[\mathrm{C}, \mathrm{O}, \mathrm{Ne} / \mathrm{H}]=0.18$ with a stable overabundance of nitrogen by 0.5 dex. The power law index between points $B$ and $C$ can vary from $\alpha=-0.3$ to $\alpha=0.1$ without significant changes in the predicted $N_{\mathrm{Ne} \text { VIII }}$, but harder spectra deliver more Ne VIII and are probably preferable. The discontinuity point $C$ and the power law index beyond it are set so as to account for measurements of AGN/QSO luminosity at $E=2 \mathrm{keV}=147 \mathrm{Ryd}$ (Steffen et al. 2006): again, metal lines available in the $z_{\mathrm{abs}}=$ 2.198 system are weakly sensitive to the coordinate of this point.

The calculations above were performed assuming gas in thermal equilibrium. However, conditions in the vicinity of the central engine vary rapidly, and gas can become overheated. Figure 4 shows the dependence of the ionization fractions of different ions on the temperature calculated for the UV spectrum from Fig. 3 (solid line) and the ionization parameter $U=0.45$ which for the solar metallicity gives the equilibrium gas temperature of $22000 \mathrm{~K}$. While the fractions of C IV and O VI decrease with rising $T$, the fraction of Ne VIII increases, i.e. the overheated gas is more abundant in Ne VIII. On the other hand, the cooling time is determined by the gas density, and, hence, the rarefied volumes - those traced by Ne VIII - remain longer in the overheated state. Thus, a fraction of Ne VIII at the value of $U$ corresponding to $[\mathrm{C} / \mathrm{O}] \sim 0$ will be higher in the cooling gas than that at the equilibrium.

To summarize, the ionizing spectrum with a sharp break in the intensity around 4 Ryd can easily provide conditions for significant amounts of C IV, O VI and Ne VIII to arise in the same absorbing cloud.

The results obtained also allow us to make some conclusions concerning the physical state of the absorbing cloud itself. Firstly, the ratio $N_{\mathrm{C} \text { IV }} / N_{\mathrm{O} \text { vI }}=20$ along with the constraint $[\mathrm{C} / \mathrm{O}] \sim 0$ and the absence (or small amount) of O IV (Fig. 1)

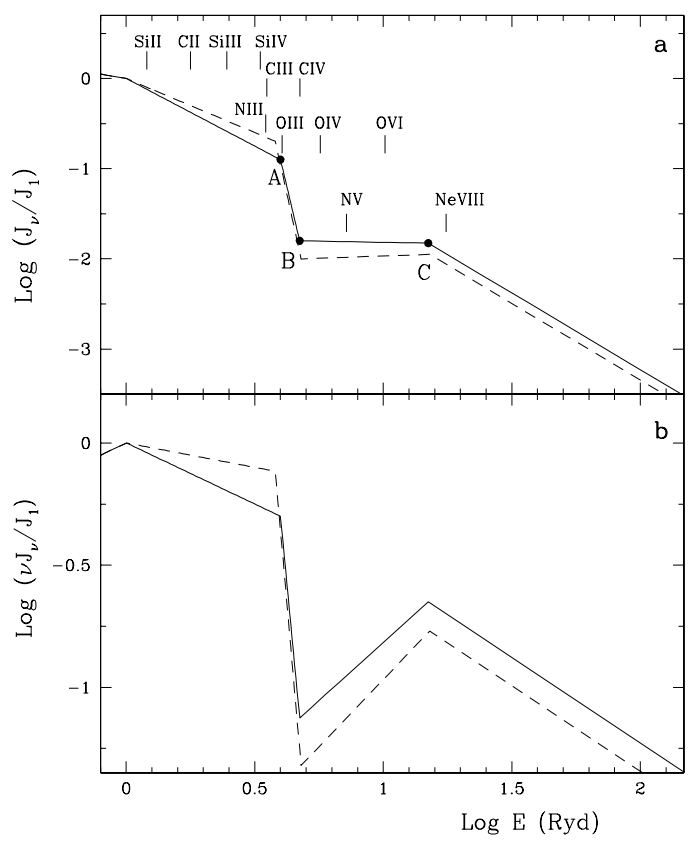

Fig. 3. Spectral energy distributions (panel a intensity, panel b flux) of the quasar radiation proposed to explain the presence of C IV, O VI, and $\mathrm{Ne}$ VIII in the associated systems towards J 2233-606. The spectra are normalized so that $J_{v}(h v=1 \mathrm{Ryd})=1$. The solid line between $1 \mathrm{Ryd}$ and point $A$ corresponds to $\alpha=1.5$, the dashed line within the same region to $\alpha=1.2$.

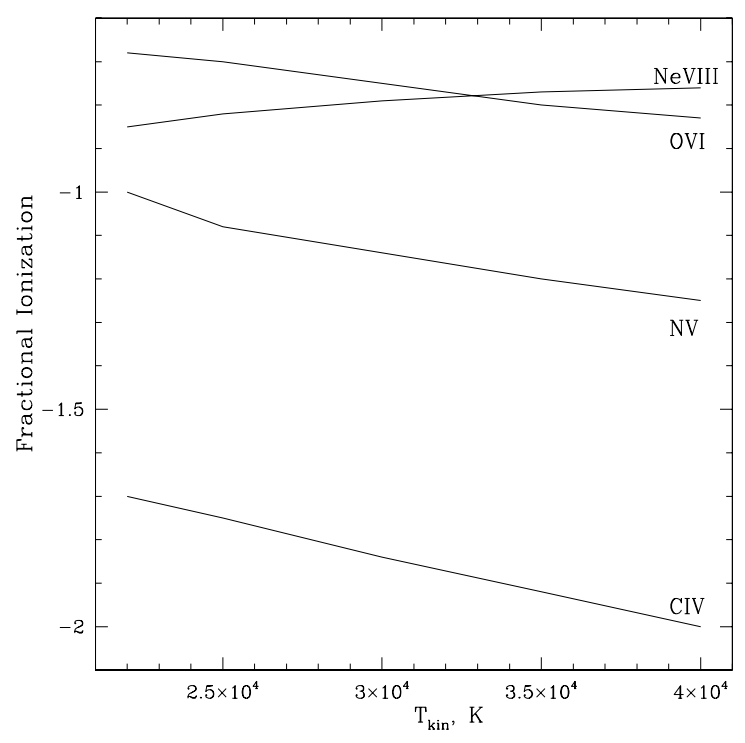

Fig. 4. Ionization fractions (in $\log _{10}$ ) of different ions versus kinetic temperature calculated for the incident ionizing spectrum shown in Fig. 3 (solid line) at the ionization parameter $U=0.45$. For the solar metallicity the equilibrium temperature is $22000 \mathrm{~K}$.

unambiguously point to photoionization as the source of the observed ionization state: in the case of collisional ionization the leading oxygen ion at the temperature which corresponds to $N_{\text {C IV }} / N_{\text {OvI }}=20$ is O IV with column density an order of magnitude larger than $N_{\text {O vI }}$ (cf., Sutherland \& Dopita 1993).

The second conclusion concerns the ion-dependent covering factors. For the system under study this dependence was stated previously by Petitjean \& Srianand (1999), and they explained this fact by different distances to the broad emission line region. However, the distribution of covering factors as shown in 


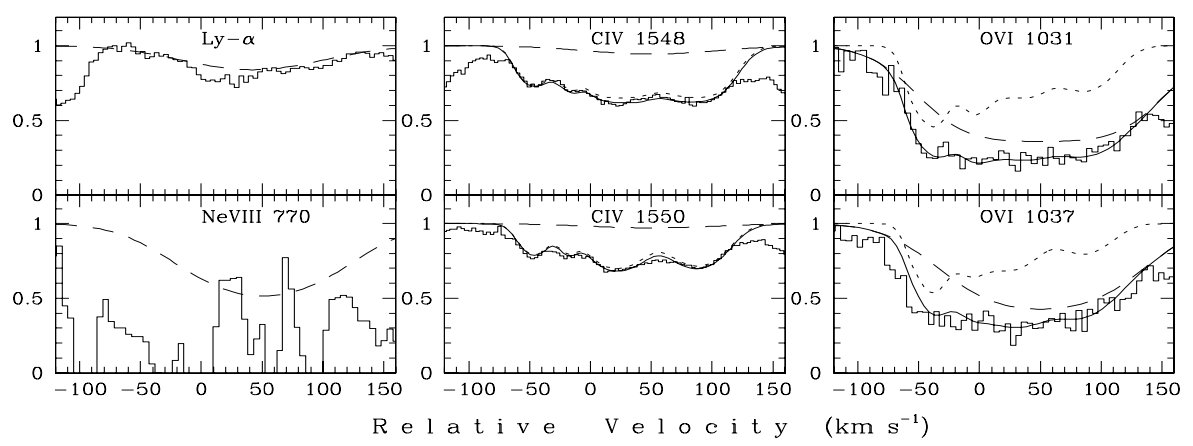

Fig. 5. Same as Fig. 1 but for the $z_{\text {abs }}=2.205$ system towards J 2233-606 (solid-line histograms). The zero radial velocity is fixed at $z=2.2050$. Synthetic profiles of C IV and O VI are produced by broad and shallow absorption (dashed curves) with a covering factor $C \sim 0.6$, and by several overlapping narrow absorptions (dotted curves) with $C \sim 0.3$. Smooth curves represent the convolution of all components according to Eq. (2). The dashed line in the Ne VIII panel is the predicted profile for the broad component corresponding to the ionizing spectrum with a break at $\sim 4$ Ryd (Fig. 3, solid line). The dashed line in the Ly- $\alpha$ panel is the predicted upper limit for the broad component.

Table 1 directly follows from a model of the associated absorption arising in a gas cloud with fluctuating density: ions of higher ionization stages trace rarefied gas which can be quite extended whereas low ionization ions originate in more dense and, hence, compact volumes. This naturally explains the increasing value of the covering factor from $\mathrm{HI}$ to O VI and the reason why the synthetic Ne VIII profile in Fig. 1 was plotted with $C_{\mathrm{Ne} \mathrm{vIII}}=0.9$.

\subsubsection{System at $Z_{\mathrm{abs}}=2.205$}

This portion of the associated absorption complex differs from the system considered above: profiles of metal doublets are more shallow and broad, column densities of C IV and O VI seem to be not very different, but the amount of Ne VIII is nevertheless significant (Fig. 5). Preliminary calculations have shown that profiles of the C IV $\lambda \lambda 1548,1550 \AA$ lines in this system cannot be fitted with a single covering factor. The winding shape of the profiles indicates that the observed absorption may be caused by several gas clouds, each with its own covering factor. For the simplest model with multiple overlapping clouds the observed intensity can be written as

$I_{v}=\prod_{k=1}^{n}\left(1-C_{i, k}+C_{i, k} \mathrm{e}^{-\tau_{k}(v)}\right)$,

where $C_{i, k}$ is the covering factor of cloud $k$ for ion $i$, and $v$ is radial velocity.

The unsaturated and unblended C IV lines can be deconvolved into separate subcomponents. Calculations give one broad component with the Doppler parameter $b=74 \mathrm{~km} \mathrm{~s}^{-1}$ and covering factor $C_{\text {broad }} \sim 0.6$ and four narrow components with $b=10-26 \mathrm{~km} \mathrm{~s}^{-1}$ and covering factors $C_{\text {narrow }}=0.2-0.35$. The column density of the broad component is $(2-4) \times 10^{13} \mathrm{~cm}^{-2}$, which is only a small fraction of the joint column density $5.5 \times$ $10^{14} \mathrm{~cm}^{-2}$ of the narrow components (Table 2). Synthetic profiles of the broad and narrow components as well as the combined line profile are plotted in Fig. 5. Since the profiles of O VI are saturated and smooth they can be deconvolved only under additional constraints. Assuming the same velocity and component structure as for the C IV doublet, we obtain the column density for the broad O VI component $\sim 1.5 \times 10^{15} \mathrm{~cm}^{-2}$ and a similar column density for the combined narrow components (Table 2). Thus, the broad component has the ratio $N_{\mathrm{O} \text { vI }} / N_{\mathrm{C} \text { IV }} \sim$ 40-70 which points to a highly ionized gas with the ionization parameter $U \sim 1$ (Fig. 2), whereas the narrow components have $N_{\mathrm{O} \text { vI }} / N_{\text {C IV }} \sim 3$ and are much less ionized with $U \sim 0.1$.
Table 2. Column densities and covering factors for the broad (b) and four narrow (n) components of the associated system $z_{\mathrm{abs}}=2.205$ towards J 2233-606 shown in Fig. 5.

\begin{tabular}{ccccc}
\hline \hline Ion & $\begin{array}{c}N_{i}, \mathrm{~cm}^{-2} \\
(\mathrm{~b})\end{array}$ & $\begin{array}{c}\sum N_{i}, \mathrm{~cm}^{-2} \\
(\mathrm{n})\end{array}$ & $\begin{array}{c}C_{i} \\
(\mathrm{~b})\end{array}$ & $\begin{array}{c}C_{i} \\
(\mathrm{n})\end{array}$ \\
\hline H I & $\lesssim 3 \mathrm{E} 13$ & & $\lessgtr 0.5$ & \\
C IV & $(2-4) \mathrm{E} 13$ & $(5.5-5.7) \mathrm{E} 14$ & 0.6 & $0.2-0.35$ \\
O VI & $(1.5-1.7) \mathrm{E} 15$ & $(1.7-1.9) \mathrm{E} 15$ & 0.65 & $0.3-0.4$ \\
Ne VIII & $(6-7) \mathrm{E} 14$ & & 0.7 & \\
\hline
\end{tabular}

With these parameters the predicted column density for Ne VIII is $\sim 6 \times 10^{14} \mathrm{~cm}^{-2}$ for the broad component (plotted in Fig. 5 for the covering factor 0.7 ) and $\sim 10^{13} \mathrm{~cm}^{-2}$ for the narrow components.

The line Nv $\lambda 1238 \AA$ is blended with Ca II $\lambda 3968 \AA$ from $z_{\text {abs }} \sim 0$ which hampers its deconvolution into components and the estimation of their covering factors. However, trial calculations with different constraints show that an overabundance of nitrogen is present in both broad and narrow components.

\subsection{NAL systems towards HE 1341-1020}

The spectrum of the quasar HE 1341-1020 was obtained with the UVES/VLT in the framework of the ESO Large Program "QSO Absorption Line Systems" (ID No. 166.A-0106). Data reduction was performed by B. Aracil.

HE 1341-1020 is a mini-BAL quasar with broad absorptions in C IV, $\mathrm{N} \mathrm{V}$ and $\mathrm{O}$ VI extending over $3000 \mathrm{~km} \mathrm{~s}^{-1}$. There is also a narrow absorption system at $z_{\mathrm{abs}}=2.147$. The quasar emission redshift estimated from the C II $\lambda 1334 \AA$ and C III $\lambda 1990] \AA$ emission lines is $z_{\mathrm{em}}=2.1485 \pm 0.002$.

\subsubsection{System at $Z_{\mathrm{abs}}=2.147$}

Ionization state and restored SED. The system at $z_{\mathrm{abs}}=2.147$ exhibits a wealth of lines of metal ions ranging from low ionization Si II, N II, Mg II up to high ionization $\mathrm{NV}$ and $\mathrm{O}$ VI (Fig. 6). The presence of many elements in different stages of ionization makes it possible to reconstruct the shape of the HE 1341-1020 spectrum in more detail than that previously described for J 2233-606. Trial calculations revealed that power law spectra as well as the AGN spectrum of Mathews \& Ferland (1987) failed to reproduce the observed ratio N II/N V resulting in the synthetic line profiles which significantly underestimate 


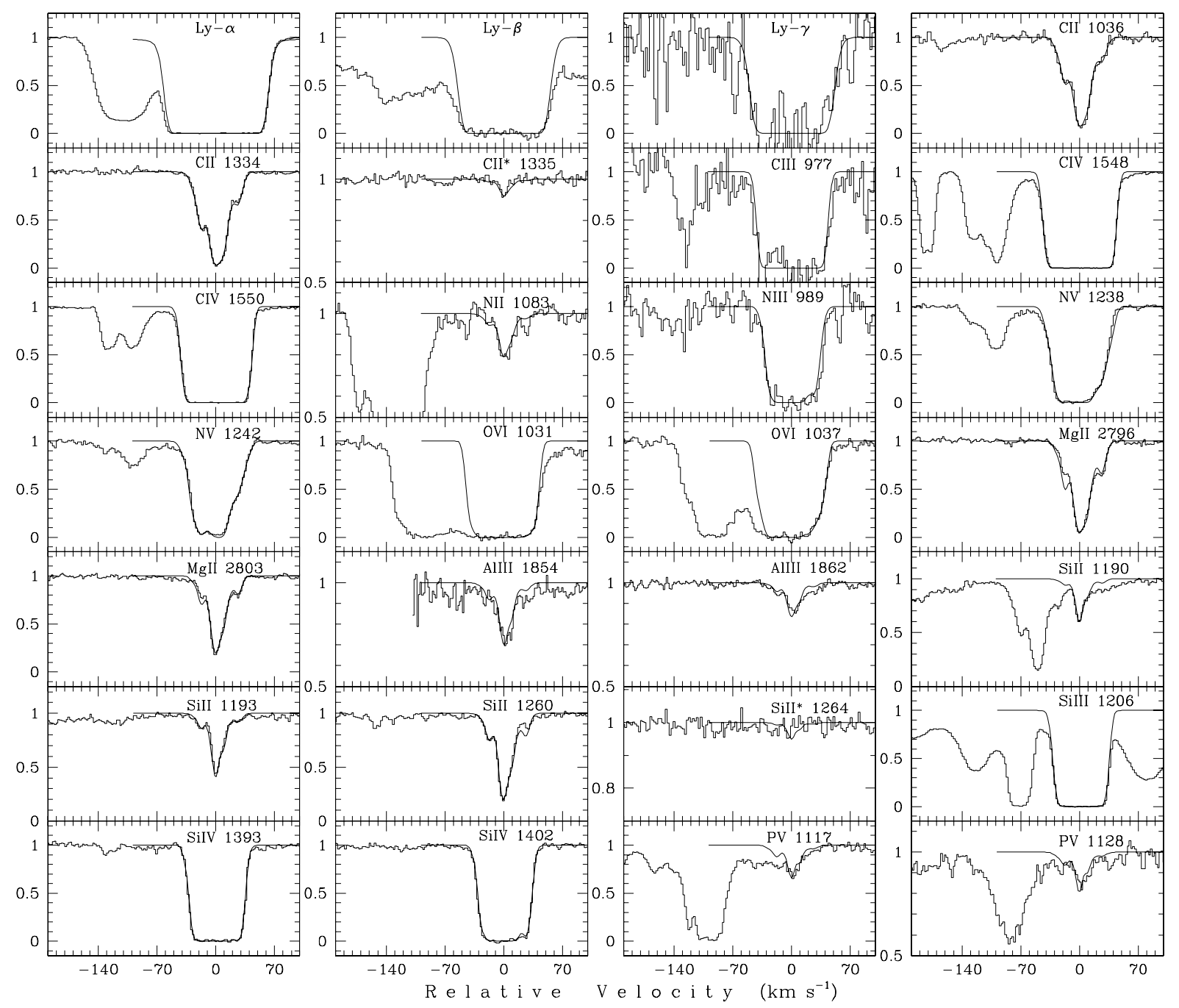

Fig. 6. Same as Fig. 1 but for the $z_{\mathrm{abs}}=2.147$ system towards HE 1341-1020 (solid-line histograms). The zero radial velocity is fixed at $z=$ 2.14736. Synthetic profiles corresponding to the ionizing spectrum with a break at $\sim 4$ Ryd (Fig. 7 ) are plotted by smooth curves. Note different vertical scales in some panels.

the observed intensity of $\mathrm{N}$ II $\lambda 1083 \AA$, and yielded relative element abundances that did not agree with existing observational and theoretical data (e.g., $[\mathrm{Mg} / \mathrm{O}] \simeq 0.7,[\mathrm{Si} / \mathrm{O}] \simeq 0.3$ ). Several types of SEDs with a break at 4 Ryd were tried as well and as a result the SED recovered from the $z_{\mathrm{abs}}=1.78$ associated system towards HE 0141-3432 (Reimers et al. 2005, Sect. 4.2) was chosen as the best initial guess for the spectral shape recovering procedure. The search for an optimal spectral shape was directed by the requirement to reproduce all observed lines under the constraint $[\mathrm{Si}, \mathrm{Mg}, \mathrm{O} / \mathrm{C}] \lesssim 0.2$. Details of calculations are given in Reimers et al. (2005, Appendix).

The resulting SED is shown in Fig. 7, and the corresponding synthetic profiles are plotted with solid curves in Fig. 6. The estimated physical parameters and column densities are given in Table 3.

As it was for the previous system, the spectral shape again has a sharp break at $\sim 4$ Ryd, but in the present case it is also possible to fix the depth of this break and the broken power law slope between 1 and 4 Ryd.

The column density of neutral hydrogen is $N_{\mathrm{HI}} \lesssim 10^{17} \mathrm{~cm}^{-2}$, i.e. the $z_{\text {abs }}=2.147$ system is a sub-LLS (optical depth in the HI Lyman continuum $\tau_{\mathrm{c}}^{\mathrm{HI}} \lesssim 0.6$ ). The predicted column density for He II is $N_{\text {He II }} \lesssim 3 \times 10^{18} \mathrm{~cm}^{-2}$ which makes the system moderately optically thick to the He II Lyman continuum $\left(\tau_{\mathrm{c}}^{\mathrm{He}}\right.$ II $\left.\simeq 4.7\right)$.

The ionizing spectrum is reconstructed from the observed absorption lines and therefore is sensitive to the local processes in the absorbing cloud itself (for more details, see Sect. 3 in Agafonova et al. 2005). In particular, for the case in question the restored quasar spectrum can be affected by the He II Lyman continuum absorption inside the cloud, i.e. the incident SED can be harder (probably by $0.2-0.3 \mathrm{dex}$ ) at energies $E>4$ Ryd than the spectrum shown in Fig. 7. The He II Lyman continuum absorption is accompanied by the recombination emission line of He II Ly- $\alpha$ (304 $\AA$ ) and by the two-photon emission which both produce a characteristic emission feature at $E \lesssim 3$ Ryd in the transmitted spectrum of the incident quasar radiation. Whether such a feature is present or not in the restored spectrum cannot be stated unambiguously because of the saturated profiles of the Si III and Si IV lines (fractions of silicon ions are most sensitive to the spectral shape of the ionizing radiation at $E \lesssim 3 \mathrm{Ryd}$ as seen in Fig. 2 in Agafonova et al. 2007).

The measured metallicity of the absorbing gas is solar (carbon) or slightly oversolar ( $\alpha$-elements) with clear underabundances of nitrogen ( 0.6 solar) and aluminium ( 0.3 solar). 


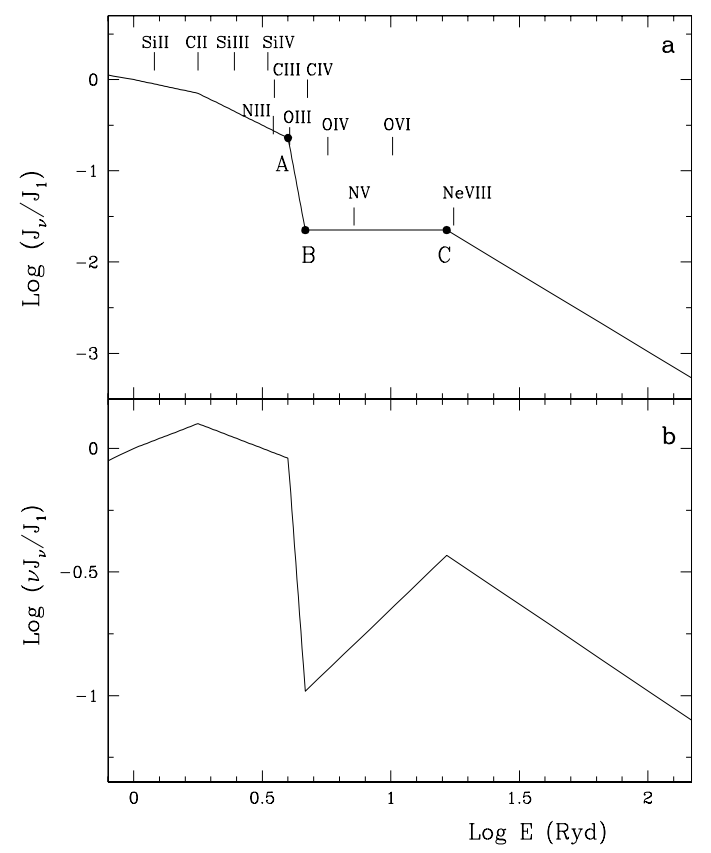

Fig. 7. SED estimated from the $z_{\mathrm{abs}}=2.147$ system towards the quasar HE 1341-1020 $\left(z_{\mathrm{em}}=2.1485\right)$.

Distance to the QSO. The presence of the ground fine-structure line $\mathrm{C} \mathrm{II}^{*} \lambda 1335 \AA$ arising from the first excited ${ }^{3} \mathrm{P}_{1}^{\mathrm{e}}$ level provides a rare opportunity to measure from the photoionization model the mean gas number density and, hence, the distance to the light source. For an ion in the interstellar (intergalactic) medium, the ratio of excited $\left(n_{2}\right)$ to ground-state $\left(n_{1}\right)$ population is equal to the ratio of the collisional excitation rate $Q_{1 \rightarrow 2}$ to the spontaneous transition probability $A_{2 \rightarrow 1}$ (Bahcall \& Wolf 1968):

$\frac{n_{2}}{n_{1}}=\frac{Q_{1 \rightarrow 2}}{A_{2 \rightarrow 1}}$.

The atomic data for $\mathrm{CII}^{*}$ are the following (Silva \& Viegas 2002): $A_{2 \rightarrow 1}=2.291 \times 10^{-6} \mathrm{~s}^{-1}$, the excitation rate by collisions with electrons at $T_{\text {kin }}=10^{4} \mathrm{~K} q_{1 \rightarrow 2}^{\mathrm{e}} \simeq 1 \times 10^{-7} \mathrm{~cm}^{3} \mathrm{~s}^{-1}$. Since collisions with other particles have much lower excitation rates, we put $Q_{1 \rightarrow 2}=q_{1 \rightarrow 2}^{\mathrm{e}} n_{\mathrm{e}}$.

From the measured column densities $N_{\mathrm{C} \text { II }}$ and $N_{\mathrm{C}_{\text {II }}^{*}}$ (see Table 3) we obtain the ratio of the mean values $n_{2}$ to $n_{1}$

$\frac{n_{2}}{n_{1}} \simeq 0.02$.

Since this system has a high degree of ionization $\left(N_{\mathrm{H}_{\mathrm{I}}} / N_{\mathrm{H}}=\right.$ $n_{\mathrm{H}^{+}} / n_{\mathrm{H}} \gg 1$, see Table 3 ), and collisions with other particles have much lower excitation rates, the total gas density equals $n_{0} \simeq 0.5 \mathrm{~cm}^{-3}$ (the contribution of the ionized helium is ignored since it has a small effect).

If this system was an intergalactic absorber, then the ionization parameter $U=0.02$ would correspond to the gas number density $n_{0} \simeq 0.001 \mathrm{~cm}^{-3}$ (assuming the intensity $J_{912}$ of the metagalactic UV background radiation at $z=2.0$ as given by Haardt \& Madau 1996). This is a clear indication that the ionizing background at the position of the $z_{\mathrm{abs}}=2.147$ system is enhanced by more than 2 orders of magnitude as compared to the intergalactic background.

In order to estimate the distance to the light source the QSO continuum luminosity at the hydrogen Lyman limit $\mathcal{L}_{v_{\mathrm{c}}}$ must be known. The intrinsic luminosity $\mathcal{L}_{v_{c}}$ can be determined
Table 3. Physical parameters of the $z_{\text {abs }}=2.147$ metal absorber towards HE 1341-1020 derived with the MCI procedure (uncertainties of the physical parameters are 10-15\%: $U_{0}$ - mean ionization parameter; $N_{\mathrm{H}}$ - total hydrogen column density; $\sigma_{\mathrm{v}}, \sigma_{\mathrm{y}}$ - dispersions of velocity and density distributions, respectively; $Z_{\mathrm{X}}=N_{\mathrm{X}} / N_{\mathrm{H}}$ - abundances of the individual elements).

\begin{tabular}{|c|c|}
\hline \multicolumn{2}{|l|}{ Parameter } \\
\hline$U_{0}$ & $1.8 \mathrm{E}-2$ \\
\hline$N_{\mathrm{H}}, \mathrm{cm}^{-2}$ & $1.8 \mathrm{E} 20$ \\
\hline$\sigma_{\mathrm{v}}, \mathrm{km} \mathrm{s}^{-1}$ & 26.6 \\
\hline$\sigma_{\mathrm{y}}$ & 0.65 \\
\hline$Z_{\mathrm{C}}$ & $2.2 \mathrm{E}-4$ \\
\hline$Z_{N}$ & $3.9 \mathrm{E}-5$ \\
\hline$Z_{\mathrm{O}}$ & $7.0 \mathrm{E}-4$ \\
\hline$Z_{\mathrm{Mg}}$ & $4.4 \mathrm{E}-5$ \\
\hline$Z_{\mathrm{Al}}$ & $8.5 \mathrm{E}-7$ \\
\hline$Z_{\mathrm{Si}}$ & $3.9 \mathrm{E}-5$ \\
\hline$Z_{\mathrm{P}}$ & $2.5 \mathrm{E}-7$ \\
\hline$\left[Z_{C}\right]^{a}$ & -0.03 \\
\hline$\left[Z_{N}\right]$ & -0.18 \\
\hline$\left[Z_{\mathrm{O}}\right]$ & 0.18 \\
\hline$\left[Z_{\mathrm{Mg}}\right]$ & 0.10 \\
\hline$\left[Z_{\mathrm{Al}}\right]$ & -0.56 \\
\hline$\left[Z_{\mathrm{Si}}\right]$ & 0.08 \\
\hline$\left[Z_{\mathrm{P}}\right]$ & 0.10 \\
\hline$N(\mathrm{H} \mathrm{I}), \mathrm{cm}^{-2}$ & $(8.6 \pm 1.5) \mathrm{E} 16$ \\
\hline$N(\mathrm{He}$ II $), \mathrm{cm}^{-2}$ & $3.0 \mathrm{E} 18^{b}$ \\
\hline$N(\mathrm{C}$ II $), \mathrm{cm}^{-2}$ & $(1.8 \pm 0.1) \mathrm{E} 14$ \\
\hline$N\left(\mathrm{C} \mathrm{II}{ }^{*}\right), \mathrm{cm}^{-2}$ & $(4.1 \pm 0.2) \mathrm{E} 12$ \\
\hline$N(\mathrm{C} \mathrm{III}), \mathrm{cm}^{-2}$ & $1.0 \mathrm{E} 12^{b}$ \\
\hline$N(\mathrm{C}$ IV $), \mathrm{cm}^{-2}$ & $(1.5 \pm 0.5) \mathrm{E} 16$ \\
\hline$N(\mathrm{~N}$ II $), \mathrm{cm}^{-2}$ & $(1.6 \pm 0.2) \mathrm{E} 13$ \\
\hline$N(\mathrm{~N}$ III $), \mathrm{cm}^{-2}$ & $2.0 \mathrm{E} 15^{b}$ \\
\hline$N(\mathrm{~N} \mathrm{v}), \mathrm{cm}^{-2}$ & $(7.1 \pm 1.5) \mathrm{E} 14$ \\
\hline$N(\mathrm{O}$ VI $), \mathrm{cm}^{-2}$ & $(3.0 \pm 0.5) \mathrm{E} 15$ \\
\hline$N(\mathrm{Mg}$ II $), \mathrm{cm}^{-2}$ & $(1.5 \pm 0.2) \mathrm{E} 13$ \\
\hline$N(\mathrm{Al} \mathrm{III}), \mathrm{cm}^{-2}$ & $(2.7 \pm 0.4) \mathrm{E} 12$ \\
\hline$N(\mathrm{Si}$ II $), \mathrm{cm}^{-2}$ & $(8.4 \pm 0.8) \mathrm{E} 12$ \\
\hline$N(\mathrm{Si} \text { II })^{*}, \mathrm{~cm}^{-2}$ & $\lesssim 2.5 \mathrm{E} 11$ \\
\hline$N(\mathrm{Si}$ III $), \mathrm{cm}^{-2}$ & $(2.7 \pm 0.5) \mathrm{E} 14$ \\
\hline$N($ Si IV $), \mathrm{cm}^{-2}$ & $(7.0 \pm 1.5) \mathrm{E} 14$ \\
\hline$N(\mathrm{Pv}), \mathrm{cm}^{-2}$ & $(6.5 \pm 0.7) \mathrm{E} 12$ \\
\hline$\langle T\rangle, \mathrm{K}$ & $1.0 \mathrm{E} 4$ \\
\hline
\end{tabular}

${ }^{a}\left[Z_{\mathrm{X}}\right]=\log \left(N_{\mathrm{X}} / N_{\mathrm{H}}\right)-\log \left(N_{\mathrm{X}} / N_{\mathrm{H}}\right)_{\odot}$.

${ }^{b}$ Calculated using the velocity and density distributions derived from hydrogen and metal profiles.

from the comparison of $V=17.1$ with the specific flux of a star having $m_{V}=0.0$ outside the Earth's atmosphere, $F_{V}^{*}=3.8 \times$ $10^{-20} \mathrm{erg} \mathrm{cm}^{-2} \mathrm{~s}^{-1} \mathrm{~Hz}^{-1}$ :

$f_{V}=10^{-0.4 \cdot 17.1} F_{V}^{*}=5 \times 10^{-27} \mathrm{erg} \mathrm{cm}^{-2} \mathrm{~s}^{-1} \mathrm{~Hz}^{-1}$.

With the approximation for the luminosity distance $d_{\mathrm{L}}$ proposed in Riess et al. (2004), one obtains $d_{\mathrm{L}} \simeq 13 \mathrm{Gpc}$ and the apparent monochromatic luminosity

$\mathcal{L}_{v}=4 \pi d_{\mathrm{L}}^{2} f_{V}(1+z)^{-1} \simeq 3 \times 10^{31} \mathrm{erg} \mathrm{s}^{-1} \mathrm{~Hz}^{-1}$,

which results in the luminosity near the Lyman limit

$\mathcal{L}_{v_{\mathrm{c}}}=\mathcal{L}_{v}\left(\frac{1750}{912}\right)^{-1} \simeq 1.5 \times 10^{31} \mathrm{erg} \mathrm{s}^{-1} \mathrm{~Hz}^{-1}$ 
Given the gas number density $n_{0}$, the distance $r$ from the QSO to the absorbing cloud can be calculated from the ionization parameter $U_{0}$ which is defined as

$U_{0}=\frac{Q\left(\mathrm{H}^{0}\right)}{4 \pi r^{2} c n_{0}}=\frac{n_{\mathrm{ph}}}{n_{0}}$,

where

$Q\left(\mathrm{H}^{0}\right)=\int_{v_{\mathrm{c}}}^{\infty} \frac{\mathcal{L}_{v}}{h v} \mathrm{~d} v$

is the number of hydrogen ionizing photons emitted per unit time by the central source.

With the Lyman continuum luminosity $\mathcal{L}_{v_{\mathrm{c}}}$ estimated above, one finds $Q\left(\mathrm{H}^{0}\right) \simeq 2 \times 10^{57}$ photons $\mathrm{s}^{-1}$, assuming $\mathcal{L}_{v}=$ $\mathcal{L}_{v_{\mathrm{c}}}\left(v / v_{\mathrm{c}}\right)^{-1}$. A substitution of the numerical values in (4) provides $r \simeq 240 \mathrm{kpc}$. Accounting for the optical depth $\tau \lesssim 0.6$ at the hydrogen ionization edge due to absorption within the cloud, the distance can be reduced to $\sim 170 \mathrm{kpc}$.

It is unclear whether an absorber at such a large distance from the light source can be gas ejected from the QSO host galaxy. However, distances as large as hundreds of kiloparsecs (mostly estimated as lower limits) are not exceptional for the associated systems (Morris et al. 1986; Tripp et al. 1996; D’Odorico et al. 2004; Reimers et al. 2005).

\subsubsection{System at $z_{\text {abs }}=2.107$}

Mini-BAL absorptions in C IV, N v and O VI at $-7000 \mathrm{~km} \mathrm{~s}^{-1}<$ $v<-1000 \mathrm{~km} \mathrm{~s}^{-1}$ are formed by a large number of overlapping components (Fig. 8) which in most cases do not allow us to estimate covering factors and, hence, to measure accurately the column densities. Fortunately, portions of the C IV $\lambda 1548 \AA$ profile between $-4100 \mathrm{~km} \mathrm{~s}^{-1}$ and $-3855 \mathrm{~km} \mathrm{~s}^{-1}\left(z_{\mathrm{abs}} \simeq 2.1066\right)$ and, correspondingly, C IV $\lambda 1550 \AA$ profile at $-3638 \mathrm{~km} \mathrm{~s}^{-1}<v<$ $-3396 \mathrm{~km} \mathrm{~s}^{-1}$ are not blended and can be used for quantitative analysis. A winding shape of the line profiles favors the model used above for the $z_{\mathrm{abs}}=2.205$ system towards J 2233-606: several narrow components seen against the broad one. Component fitting according to Eq. (2) gives for the broad component the column density $N_{\mathrm{C} \text { Iv, broad }}=4.2 \times 10^{13} \mathrm{~cm}^{-2}$ and the covering factor $C_{\mathrm{C} \text { Iv, broad }}=0.8$, and for the four narrow components the total column density $N_{\mathrm{C} \text { Iv,narrow }}=5.8 \times 10^{13} \mathrm{~cm}^{-2}$ and the covering factor $0.16 \lesssim C_{\mathrm{C}_{\text {IV }} \text {,narrow }} \lesssim 0.36$ (thin smooth and dotted curves in panel C IV in Fig. 8).

From the doublets N V and O VI only the lines N v $\lambda 1238 \AA$ and O VI $\lambda 1031 \AA$ are available. Assuming for N V similar velocity structure and covering factors as calculated for C IV, one obtains for the broad $\mathrm{N} \mathrm{V}$ component the column density $N_{\mathrm{N} \text { v,broad }}=(1.4-1.6) \times 10^{14} \mathrm{~cm}^{-2}$. The saturated O VI $\lambda 1031 \AA$ line indicates the covering factor of $C_{\mathrm{OvI} \text {,broad }} \simeq 0.90-0.95$, which gives the upper limit for the column density of the broad O VI component $N_{\mathrm{O} \text { vI,broad }}<2 \times 10^{15} \mathrm{~cm}^{-2}$.

The ratio $N_{\mathrm{O} \text { vi,broad }} / N_{\mathrm{C} \text { Iv, broad }} \lesssim 50$ together with the assumption of solar relative abundances $[\mathrm{C} / \mathrm{O}] \sim 0$ lead to the range of the ionization parameters $U$ which translate the column densities of $\mathrm{C} \mathrm{IV}$ and $\mathrm{N} \mathrm{V}$ observed in the broad component into the reliable relative overabundance of nitrogen $[\mathrm{N} / \mathrm{C}]>0$ for both a power law SED and a SED with a discontinuity at 4 Ryd. Thus, the gas comprising the broad component in the mini-BAL absorption at the radial velocity $v \simeq-4000 \mathrm{~km} \mathrm{~s}^{-1}$ obviously differs from the gas producing the narrow line absorption at $z_{\mathrm{abs}}=2.14736$ (Fig. 6) where the nitrogen underabundance of $[\mathrm{N} / \mathrm{C}]=-0.15$ was detected. This will be discussed further in Sect. 3.2.

\subsection{NAL systems towards HE 2347-4243}

An associated complex towards the quasar HE 2347-4243 $\left(z_{\mathrm{em}}=\right.$ 2.902) consisting of many absorption systems and extending over $1500 \mathrm{~km} \mathrm{~s}^{-1}$ was described in detail in Fechner et al. (2004). It was found that column densities of ions measured in certain systems favored the ionizing spectrum with a break at $E \sim$ 4 Ryd. However, an exact spectral shape in the range $E>1$ Ryd was not estimated. Below we re-analyze a few systems from this complex with the objective to reach more tenable conclusions about the SED of HE 2347-4243.

\subsubsection{System at $z_{\mathrm{abs}}=2.898$}

This system (component 8 in Fechner et al. 2004) exhibits strong lines of the doublets $\mathrm{CIV}, \mathrm{NV}$ and $\mathrm{OVI}$ which allow us to estimate accurately the covering factors and column densities (Fig. 9). There is a weak hydrogen absorption Ly- $\alpha$, whereas the observed intensity at the position of Ly- $\beta$ may be due to a blend with some forest line. However, these lines can be used to set a limit on the covering factor for H I. The C III $\lambda 977 \AA$ line is also observed, albeit blended.

The measured column densities and covering factors for C IV, N V and O VI ions are given in Table 4. The apparent intensity at the position of $\mathrm{Ly}-\beta$ constrains the covering factor and column density for $\mathrm{HI}$ : $C_{\mathrm{H}_{\mathrm{I}}}<0.6$, and $N_{\mathrm{H}_{\mathrm{I}}}<2.3 \times 10^{13} \mathrm{~cm}^{-2}$. The covering factor for $\mathrm{C}$ III can lie between $C_{\mathrm{HI}_{\mathrm{I}}}$ and $C_{\mathrm{CIV}}$, i.e. $0.60<C_{\mathrm{C}_{\text {III }}}<0.97$. The observed intensity at the expected position of the $\mathrm{C}$ III line allows us to estimate the upper limits to its column density: $N_{\mathrm{C}_{\text {III }}}<9.0 \times 10^{12} \mathrm{~cm}^{-2}$ if $C_{\mathrm{C} \text { III }}=0.60$, and $N_{\mathrm{C} \text { III }}<4.7 \times 10^{12} \mathrm{~cm}^{-2}$ if $C_{\mathrm{C} \text { II }}=0.97$.

Taking the ratio $N_{\mathrm{C} \text { IV }} / N_{\mathrm{O} \text { VI }}=2.3$ and assuming solar relative abundance of carbon to oxygen, $[\mathrm{C} / \mathrm{O}] \sim 0$, we can estimate the ionization parameter $U$ for a given SED. The limits set above on $N_{\mathrm{C} \text { III }}$ reject unambiguously any type of a power law spectrum as well as the AGN spectrum of Mathews \& Ferland (1987): for $U$ corresponding to $[\mathrm{C} / \mathrm{O}] \sim 0$ these spectra significantly overpre$\operatorname{dict} N_{\mathrm{C} \text { III }}$.

In order to comply with the limits on $N_{\mathrm{C} \text { III }}$ the ionizing spectrum should be quite hard at $1<E<4$ Ryd $(\alpha<1.0)$ and have a sharp break at $E \sim 4$ Ryd with an intensity depression by more than an order of magnitude. The spectrum of HE 2347-4243 is known up to $\sim 350 \AA$ (2.6 Ryd) and it indeed shows a very hard continuum in the EUV range with $\alpha=0.56$ (Reimers et al. 1998; Fechner et al. 2004). Adopting this index, we can estimate the depth of the intensity break at 4 Ryd: $J_{\mathrm{A}} / J_{\mathrm{B}}=20$ (assuming $\left.C_{\mathrm{C} \text { III }}=0.60\right)$, and $J_{\mathrm{A}} / J_{\mathrm{B}}=30$ (assuming $C_{\mathrm{C} \text { III }}=0.97$ ).

With these EUV spectra, the carbon and oxygen abundances in the $z_{\text {abs }}=2.898$ system can vary between $5-15$ solar - depending on the adopted covering factor for $\mathrm{H}$ I and the depth of the continuum break at 4 Ryd, but nitrogen remains always underabundant: $[\mathrm{N} / \mathrm{O}, \mathrm{C}]<0$. Absolute values of the abundances are uncertain not only due to the unknown covering factor of $\mathrm{HI}$, but also because of a possible overionization of $\mathrm{H}$ I as a result of high temperature: calculations with CLOUDY shows that the $\mathrm{H}$ I fraction decreases more rapidly than the fractions of C IV and $\mathrm{O}$ VI when the temperature rises above the thermal equilibrium level. On the other hand, the time to reach the ionization equilibrium is longer for H I than for other ions (Osterbrock 1974). This means that calculations that use the H I fraction corresponding to the thermal equilibrium can significantly underpredict the total hydrogen amount and deliver an artificially boosted metallicity. In principle, a neighboring system centered at $-415 \mathrm{~km} \mathrm{~s}^{-1}$ 

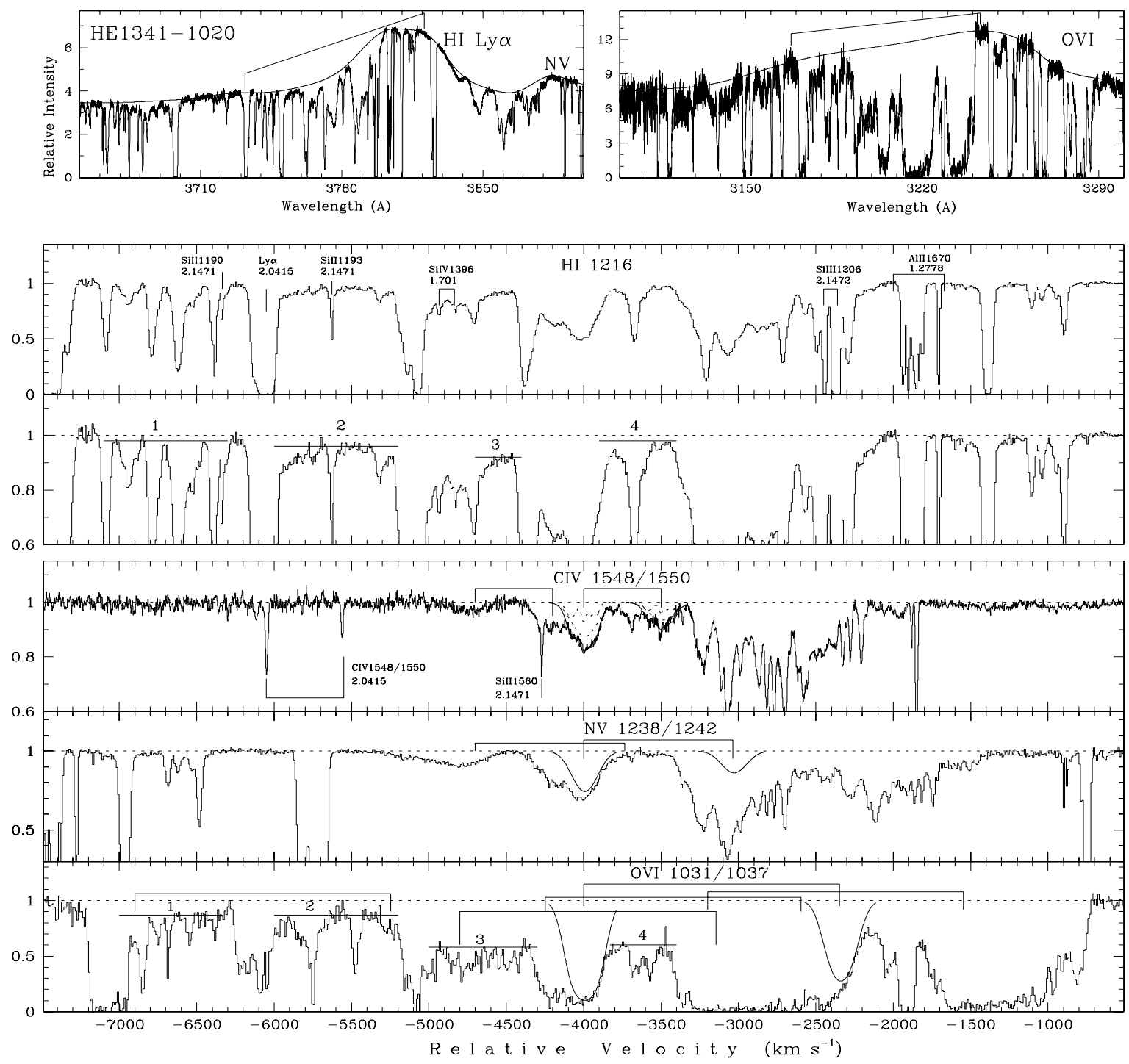

Fig. 8. Mini-BAL absorptions towards HE 1341-1020. The two upper panels represent portions of the original QSO spectrum in the vicinity of broad emission lines H I Ly- $\alpha$ and O VI + H I Ly- $\beta$. The smooth curves are the local continua calculated through the spline interpolation between "clear" continuum windows seen at high spectral resolution $\left(F W H M \simeq 6 \mathrm{~km} \mathrm{~s}^{-1}\right)$ in the UVES/VLT data. The lower panels show the zoomed portions marked by long brackets in the upper panels. The vertical axis is normalized intensity. The zero radial velocity is fixed at $z_{\mathrm{em}}=2.1485$. Two combined panels with H I $\lambda 1216$ profiles are, respectively, the general view with labeled absorption lines identified at different redshifts and the same but zoomed in the vertical scale spectrum to illustrate broad and shallow absorption features (marked by numbers over horizontal lines) caused by H I Ly- $\alpha$ arising from a fast gas outflow. The corresponding broad and shallow features are seen in the O VI panel. Absorption centered at $v=-4000 \mathrm{~km} \mathrm{~s}^{-1}\left(z_{\mathrm{abs}}=2.107\right)$ can be deconvolved into one broad and shallow component and several overlapping narrow components (shown by dotted curves). Smooth curves show C IV - the synthetic profile of the convolved broad and narrow absorptions; N V - the broad component corresponding to the covering factors and the velocity structure as determined from C IV; O VI - the limiting profile for the broad component. Long brackets in the three bottom panels mark components of the corresponding doublets to illustrate their strong overlapping.

(Fig. 9) may be considered as support for this statement: although accurate calculations are prevented by line blending, an upper limit for the gas metallicity does not exceed 3 solar values. Unfortunately, it is impossible to distinguish between two interpretations of absorption lines seen at $v<-325 \mathrm{~km} \mathrm{~s}^{-1}$ : whether they are caused by the overlapping of clouds with different metallicities or by clouds with similar metallicity but some of them not in thermal equilibrium.

\subsubsection{System at $z_{\mathrm{abs}}=2.901$}

This system (centered at $v \simeq-85 \mathrm{~km} \mathrm{~s}^{-1}$ in Fig. 9, component 10 in Fechner et al. 2004), exhibits unblended weak lines of the doublets $\mathrm{CIV}$ and $\mathrm{NV}$ along with strong saturated lines of OVI partially blended in the wings. The line H I Ly- $\alpha$ is saturated and black at the center which means that the absorber completely covers the light source. Assuming constant metallicity across the cloud, it is possible to reconstruct the line profiles from their unblended parts. The obtained column densities are given in Table 4. A spectral feature at $v \simeq-25 \mathrm{~km} \mathrm{~s}^{-1}$ in the $\mathrm{O}$ VI profile is due to the assumption of constant metallicity and is uncertain since the corresponding parts in both $\mathrm{O}$ VI lines are blended. However, its input to the total column density of $\mathrm{O}$ VI is insignificant because $N_{\mathrm{O} \text { vI }}$ is mostly determined by the central portion of the $\mathrm{O}$ VI lines which are clear.

The measured ratio $N_{\mathrm{O} \text { vI }} / N_{\mathrm{C} \text { IV }}=200$ is extremely high and points to an ionizing radiation much stronger than the intergalactic level: for the intergalactic absorbers with similar 

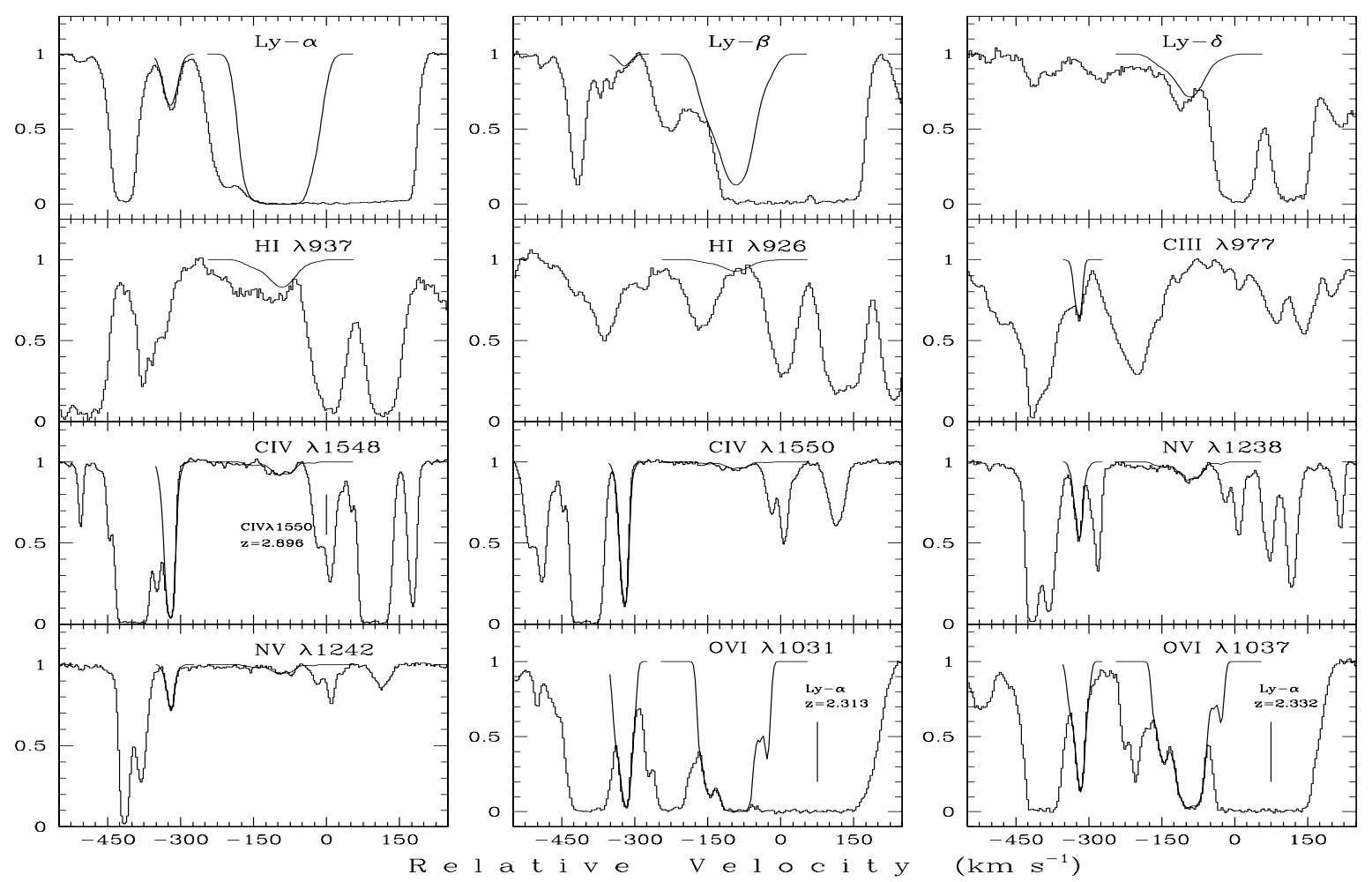

Fig. 9. Same as Fig. 1 but for the $z_{\text {abs }}=2.898\left(v \simeq-317 \mathrm{~km} \mathrm{~s}^{-1}\right)$ and $z_{\text {abs }}=2.901\left(v \simeq-85 \mathrm{~km} \mathrm{~s}^{-1}\right)$ systems towards HE $2347-4243$ (solid-line histograms). The zero radial velocity is fixed at $z=2.9021$. Synthetic profiles corresponding to the ionizing spectrum with a break at $\sim 4$ Ryd (Fig. 10) are plotted by smooth curves. See text for details.

Table 4. Column densities and covering factors for the associated systems at $z_{\text {abs }}=2.898$ and 2.901 towards HE 2347-4243 shown in Fig. 9.

\begin{tabular}{|c|c|c|c|c|}
\hline & \multicolumn{2}{|l|}{$z=2.898$} & \multicolumn{2}{|l|}{$z=2.901$} \\
\hline Ion & $N, \mathrm{~cm}^{-2}$ & $C$ & $N, \mathrm{~cm}^{-2}$ & $C$ \\
\hline \multirow[t]{2}{*}{$\mathrm{HI}$} & $2.3 \mathrm{E} 13$ & 0.6 & $(7.6 \pm 1.0) \mathrm{E} 14$ & 1.0 \\
\hline & $1.1 \mathrm{E} 13$ & 0.97 & & \\
\hline \multirow[t]{2}{*}{ C III } & $9.0 \mathrm{E} 12$ & 0.6 & & \\
\hline & $4.8 \mathrm{E} 12$ & 0.97 & & \\
\hline CIV & $(1.2 \pm 0.1) \mathrm{E} 14$ & 0.97 & $(6.7 \pm 1.0) \mathrm{E} 12$ & 1.0 \\
\hline $\mathrm{NV}$ & $(2.6 \pm 0.2) \mathrm{E} 13$ & 0.97 & $(1.5 \pm 0.3) \mathrm{E} 13$ & 1.0 \\
\hline O VI & $(2.8 \pm 0.2) \mathrm{E} 14$ & 0.985 & $(1.3 \pm 0.2) \mathrm{E} 15^{a}$ & 1.0 \\
\hline
\end{tabular}

${ }^{a}$ Both O VI $\lambda \lambda 1031,1037 \AA$ lines are blended in the red wings which are restored from $v$ - $d$ distributions obtained from other metal lines and assuming constant metallicity within the absorber. The column density is set by the portion $-60<v<40 \mathrm{~km} \mathrm{~s}^{-1}$ which is unblended.

column densities of $\mathrm{HI}$, the ratio $N_{\mathrm{OVI}} / N_{\mathrm{CIV}}$ is about $10-13$ (Reimers et al. 2006). Thus, the system under study is probably located close to the quasar.

With the same ionizing spectrum as was determined for the $z_{\mathrm{abs}}=2.898$ system, the ionization parameter $U$ can be estimated if the relative abundance of carbon to oxygen $[\mathrm{C} / \mathrm{O}]$ is known. The condition $[\mathrm{C} / \mathrm{O}] \sim 0$ used to fix $U$ in the preceding subsections cannot be fulfilled in the present case: starting at $U \sim$ 1 the ionization curves of $\mathrm{O}$ VI and $\mathrm{C}$ IV are almost parallel giving the ratio of the ion fractions $\lg \left(\Upsilon_{\mathrm{OVI}} / \Upsilon_{\mathrm{CIV}}\right)=0.8$ and thus producing a stable overabundance of oxygen $[\mathrm{O} / \mathrm{C}] \sim 0.2$. At lower $U(\lesssim 1)$ the overabundance of oxygen increases.

From measurements in the Galactic and extragalactic $\mathrm{H}$ II regions it is known that low-metallicity gas can be overenriched in oxygen with the safe upper bound $[\mathrm{O} / \mathrm{C}]<0.5$ (Henry et al. 2000; Nava et al. 2007). This gives $U>0.2$ and a metal content relative to solar $[\mathrm{C}]<-1.0,[\mathrm{O}]<-0.5,[\mathrm{~N}]<-1.2$. These relative abundances may indicate $\mathrm{SNe}$ II explosions as a main source of metal enrichment in the $z_{\mathrm{abs}}=2.901$ absorber.

The metal content of the absorbing gas redward of the $z_{\text {abs }}=2.901$ absorber (systems centered at $v=0 \mathrm{~km} \mathrm{~s}^{-1}$ and $v=115 \mathrm{~km} \mathrm{~s}^{-1}$ in Fig. 9) is uncertain due to blending of both $\mathrm{O}$ VI lines with forest absorptions. However, the column density of neutral hydrogen can be estimated quite accurately from the break at $912 \AA$ (rest frame) clearly seen in the spectrum of HE 2347-4243: $N(\mathrm{HI})=(1.6-2) \times 10^{16} \mathrm{~cm}^{-2}$. Depending on the ionization parameter of the gas and the spectral shape of the incident ionizing radiation the corresponding amount of He II lies in the interval $(2-5) \times 10^{17} \mathrm{~cm}^{-2}$ and, thus, He II can soften the transmitted UV spectrum by $0.3-0.4 \mathrm{dex}$ at $E>4 \mathrm{Ryd}^{1}$. Since the relative location of the absorbers along the line of sight from the quasar towards the observer is unknown, it cannot be excluded that the absorber at $z_{\mathrm{abs}}=2.898$ follows in physical space after the absorbers considered here. This means that the SED reconstructed from the $z_{\mathrm{abs}}=2.898$ system may represent the quasar radiation softened partly by the intervening gas seen at $-150 \mathrm{~km} \mathrm{~s}^{-1}<v<150 \mathrm{~km} \mathrm{~s}^{-1}$.

\subsection{System at $Z_{a b s}=2.352$ towards Q0329-385}

The spectrum of the quasar Q 0329-385 was obtained with the UVES/VLT in the framework of the ESO Large Program "QSO Absorption Line Systems" (ID No. 166.A-0106). Data reduction was performed by B. Aracil. The system at $z_{\mathrm{abs}}=2.352$ was first

\footnotetext{
1 Smette et al. (2002) suggest for this system $N(\mathrm{He}$ II) $\gtrsim 2.4 \times$ $10^{18} \mathrm{~cm}^{-2}$, which is a clear overestimation: the spectrum of HE 2347 4243 which is extremely hard at $1<E<4$ Ryd cannot provide $\eta=N(\mathrm{He}$ II $) / N(\mathrm{H} \mathrm{I})>100$ for any value of the break at 4 Ryd.
} 
described in Bergeron et al. (2002) in their study of intergalactic O VI absorbers and recently by Schaye et al. (2007).

The redshift of the quasar Q $0329-385$ is $z_{\mathrm{em}}=2.435$ (H I Ly$\alpha$ ) which means that the $z_{\mathrm{abs}}=2.352$ system is detached from the QSO by $\sim 7400 \mathrm{~km} \mathrm{~s}^{-1}$. There are clear lines of the doublet C IV, whereas doublets $\mathrm{NV}$ and O VI are blended, each in one component (Fig. 11). The relative intensities of both $\mathrm{C}$ IV lines do not show any evidence of incomplete coverage of the light source. The system is very much like that at $z_{\mathrm{abs}}=2.898$ towards HE 2347-4243 (Sect. 2.3.1): strong lines of highly ionized metals combined with a weak $\mathrm{H}$ I line.

The measured column densities are given in Table 5. The present system shows the column density ratio $N_{\mathrm{O} \text { vI }} / N_{\mathrm{C} \text { IV }}=3.7$ which is only slightly higher than $N_{\mathrm{OVI}} / N_{\mathrm{C} \text { IV }}=2.3$ of the $z_{\mathrm{abs}}=$ 2.898 system, but it contains much more nitrogen: $N_{\mathrm{Nv}} / N_{\mathrm{C} \text { IV }}=$ 1.34 compared to $N_{\mathrm{Nv}} / N_{\mathrm{C} \text { IV }}=0.21$ at $z_{\mathrm{abs}}=2.898$.

The apparent profile of Ly- $\alpha$ is inconsistent with the assumption of constant metallicity throughout the absorber (synthetic profiles are shown by the smooth curves in Fig. 11) ${ }^{2}$. The H I Ly$\beta$ line is blended and can be employed merely to set a lower limit for the covering factor and, correspondingly, an upper limit for $N(\mathrm{HI})$. Since the C III $\lambda 977 \AA$ line is blended with a strong line C IV $\lambda 1548 \AA$ from the $z_{\text {abs }}=1.115$ system, the only way to fix the ionization parameter is to use the condition $[\mathrm{C} / \mathrm{O}] \sim 0$.

With this restriction, any reasonable spectral index $\alpha$ of a pure power law SED $(\alpha=1.0-1.8)$ leads to $\mathrm{C}$ and $\mathrm{O}$ abundances of 10-20 solar and to a nitrogen abundance of 20-40 solar. These values are too high, even if one assumes that hydrogen is overheated (in fact, there are no signs of overheating in this system). On the other hand, for the ionizing spectra with a break at 4 Ryd the condition [C/O] $\sim 0$ is fulfilled at higher $U$ than for the power law spectra, resulting in a lower fraction of neutral hydrogen and, hence, a higher amount of total hydrogen, whereas the fractions of C IV and O VI do not change. Thus, the metal abundances decrease: the softer the spectrum at $E>4$ Ryd the lower the metallicity. For example, the spectrum restored for the $z_{\mathrm{abs}}=2.898$ system (Fig. 10, solid line) gives $[\mathrm{C}]=[\mathrm{O}]=0.5$ and $[\mathrm{N}]=0.9$. The accurate value of the continuum depression at 4 Ryd cannot be estimated since the real metallicity of the gas cloud at $z_{\mathrm{abs}}=2.352$ is of course unknown. However, we can conclude that the SED with a break at 4 Ryd is clearly preferable for this system. Another conclusion is a revealed significant relative overabundance of nitrogen, $[\mathrm{N} / \mathrm{C}] \sim 0.3-0.4$, which was constantly reproduced for any ionizing spectrum tried.

\section{Discussion}

\subsection{The origin of the break at 4 Ryd}

All NAL systems considered above as well as NAL systems towards HE 0141-3932 (Reimers et al. 2005) are best described with ionizing spectra having a break at $E \approx 4$ Ryd. It seems natural to relate this break to the Lyman continuum absorption in He II. Then the question arises: where does this absorption occur?

Firstly, this absorption can be produced by the NAL systems themselves - by those with $N(\mathrm{HI})>10^{16} \mathrm{~cm}^{-2}$. This possibility was suggested in Reimers et al. (1997) and then discussed also in Smette et al. (2002) and Shull et al. (2004). Among considered absorbers, the system at $z_{\text {abs }}=1.7103$ towards HE 0141-3932 (Reimers et al. 2005), and systems

2 The velocity shift between the dominant component in $\mathrm{H}$ I and metal lines also was noticed for the $z_{\mathrm{abs}}=2.352$ system by Shaye et al. (2007).
Table 5. Column densities and covering factors for the associated system at $z_{\text {abs }}=2.352$ towards Q 0329-385 shown in Fig. 11 .

\begin{tabular}{ccc}
\hline \hline Ion & $N, \mathrm{~cm}^{-2}$ & $C$ \\
\hline $\mathrm{H} \mathrm{I}$ & $(1.3-1.4) \mathrm{E} 13^{a}$ & 0.6 \\
& $(7-8) \mathrm{E} 12^{a}$ & 1.0 \\
$\mathrm{C}$ III & $3.6 \mathrm{E} 12^{b}$ & 1.0 \\
$\mathrm{C}$ IV & $(3.2 \pm 0.1) \mathrm{E} 13$ & 1.0 \\
$\mathrm{~N}$ III & $2.9 \mathrm{E} 12^{b}$ & 1.0 \\
$\mathrm{~N} \mathrm{~V}$ & $(4.0 \pm 0.2) \mathrm{E} 13$ & 1.0 \\
O VI & $(1.2 \pm 0.1) \mathrm{E} 14$ & 1.0 \\
\hline
\end{tabular}

${ }^{a}$ The red wing of the apparent profile inconsistent with the assumption of constant metallicity across the absorber. The synthetic profile is restored on the basis of velocity-density $(v-d)$ distributions obtained from metal lines and assuming homogeneous metallicity.

${ }^{b}$ Estimated from $v$ - $d$ distributions obtained from the metal lines C IV, $\mathrm{N}$ V, O VI and SED shown in Fig. 10.

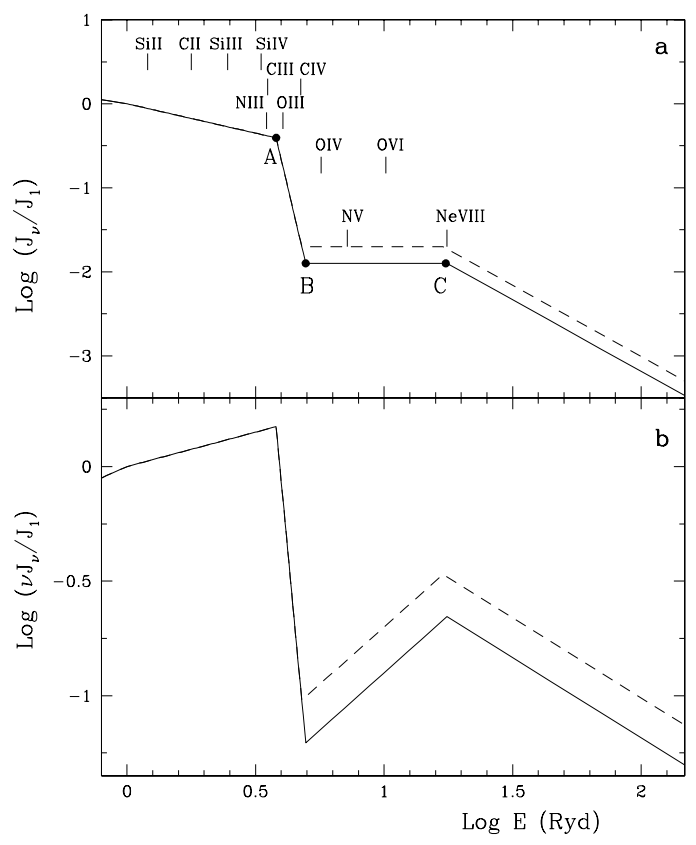

Fig. 10. SED restored from the $z_{\text {abs }}=2.898$ system towards the quasar HE 2347-4243 $\left(z_{\mathrm{em}}=2.902\right)$. The dashed line represents the ionizing spectrum corresponding to the covering factor $C_{\mathrm{C} \text { III }}=0.6$, solid line to $C_{\mathrm{CIII}}=0.97$ (see text for details).

at $z_{\mathrm{abs}}=2.901(\mathrm{HE} 2347-4243)$ and $z_{\mathrm{abs}}=2.147(\mathrm{HE} 0141-$ 3932) from the present work belong to this group. The $z_{\mathrm{abs}}=$ 2.147 system with $N(\mathrm{HI}) \approx 10^{17} \mathrm{~cm}^{-2}$ and $N($ He II $) \approx 3 \times$ $10^{18} \mathrm{~cm}^{-2}$ can noticeably soften the incident radiation due to internal absorption in the He II Lyman continuum, but the metal lines detected show that the incident radiation should already have a significant break at $E=4$ Ryd (Sect. 2.2.1). The $z_{\mathrm{abs}}=$ 1.7103 and $z_{\text {abs }}=2.901$ systems have $N($ He II $)<5 \times 10^{17} \mathrm{~cm}^{-2}$ and also cannot account for the whole depth of the break at 4 Ryd in the reconstructed spectra. Besides, lines of sight towards J 2233-606 and Q 0329-385 with the associated systems at $z_{\mathrm{abs}}=2.198$ (Sect. 2.1.1) and $z_{\mathrm{abs}}=2.352$ (Sect. 2.4), both strongly favoring the ionizing spectrum broken at 4 Ryd, do not show any absorption with $N(\mathrm{HI}) \sim 10^{16} \mathrm{~cm}^{-2}$ in the range $z_{\mathrm{em}}-z<0.3$. Thus, in general the $z_{\mathrm{abs}} \approx z_{\mathrm{em}}$ systems are unlikely to be the main source of the observed He II Lyman continuum absorption. 

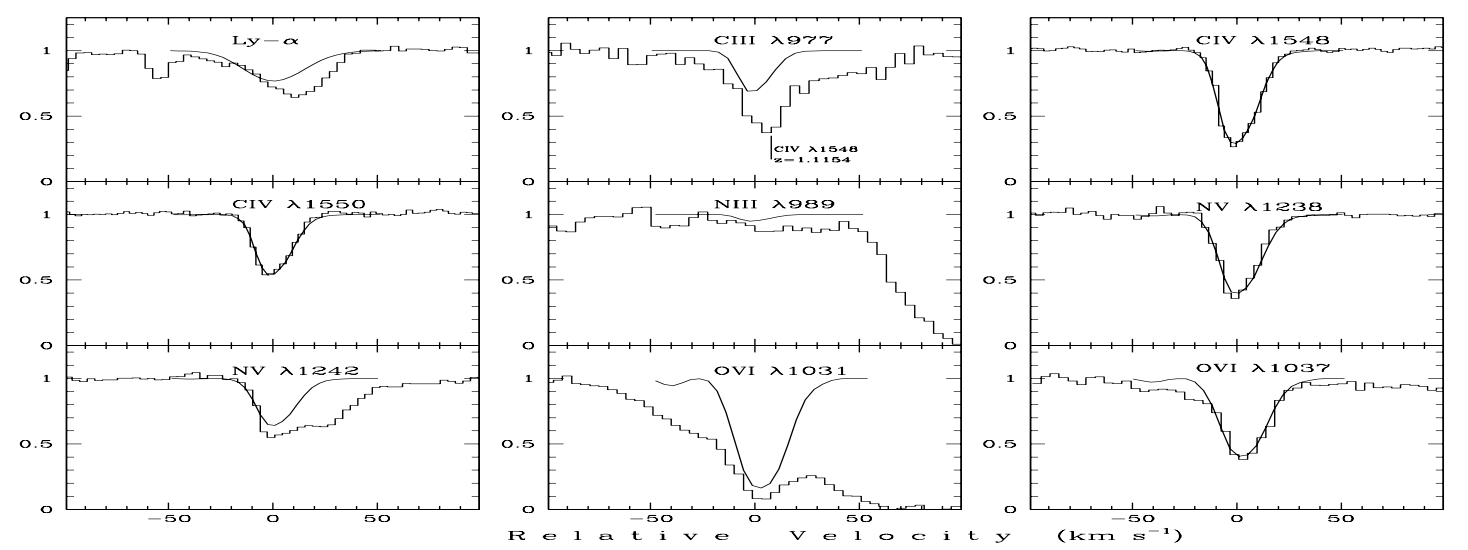

Fig. 11. Same as Fig. 1 but for the $z_{\text {abs }}=2.352$ system towards Q 0329-385 (solid-line histograms). The zero radial velocity is fixed at $z=2.35205$. Synthetic profiles are plotted by smooth curves.

More probably, the bulk of the 4 Ryd discontinuity is caused by the He II absorption in quasar winds which show a common occurrence both in quasars and AGNs. The accretion disk winds are supposed to be accelerated by the combination of radiation pressure and magneto-centrifugal forces and comprise dense gas near the equator seen as UV broad absorption lines (BAL) and highly ionized shielding gas which protects the inner wind regions from overionization by X-rays from the central source (Murray et al. 1995; Proga et al. 2000; Everett 2005). All quasars considered here do not belong to BAL quasars (only HE 0141-3932 can be classified as mini-BAL). The question is whether their spectra contain any features that could allow us to conclude that the wind absorption is indeed present and that the column density of He II in the outflowing gas is of the order of $\sim 10^{18} \mathrm{~cm}^{-2}$ (the depth of the 4 Ryd break $\sim 1 \mathrm{dex}$ ).

Model calculations with CLOUDY show that for any incident ionizing spectrum the ratio $\eta=N(\mathrm{He}$ II $) / N(\mathrm{HI})$ first increases with the rise of the ionization parameter $U$, but attains a constant value when $U$ becomes large (Fig. 19 in Agafonova et al. 2007). For power law spectra this ratio equals 20-40 depending on the slope of the spectrum (the steeper the spectrum the larger $\eta$ ) and remains almost constant at $U>0.1$. Thus, $N(\mathrm{He}$ II $)$ of $\sim 10^{18} \mathrm{~cm}^{-2}$ corresponds to $N(\mathrm{HI})$ of a few times $10^{16} \mathrm{~cm}^{-2}$. The ionization parameter $U$ can be constrained if we assume the total column density of hydrogen $N(\mathrm{H}) \sim$ $10^{22}-10^{23} \mathrm{~cm}^{-2}$ which is estimated from X-ray observations of quasars (Murray et al. 1995; Piconcelli et al. 2005). This gives $U \sim 1-5$. In this $U$ range, among ions observable in the UV the highest fractions exhibit O VI (1/30-1/100 of all oxygen) and Ne VI, Ne VII, Ne VIII (1/4-1/10 of all neon) whereas the ionization fraction of C IV is negligible. In a UV quasar spectrum, gas with such parameters is expected to leave footprints seen as H I Ly- $\alpha \lambda 1215 \AA$ absorption, H I break at $\lambda \leq 912 \AA$ and - in the case of high metallicity - O VI $\lambda \lambda 1032,1037 \AA$, Ne VI $\lambda \lambda 401,430 \AA$, Ne VII $\lambda 465 \AA$, and Ne VIII $\lambda \lambda 770,780 \AA$ absorption features. Since the gas velocity range in the accretion disk winds exceeds $10000 \mathrm{~km} \mathrm{~s}^{-1}$, these absorption features should look like wide and shallow troughs, and a break at the hydrogen ionization edge as a slow continuous depression towards higher frequencies. In the given $U$ range the oxygen exists mostly in the form of O VII (almost $90 \%$ of all oxygen) and $\mathrm{O}$ VIII ( $\sim 10 \%$ of oxygen), i.e. is best accessible in the X-ray region.

We do find the expected features in the quasar spectra considered in the present work. The depression at $\lambda \leq 912 \AA$ (rest-frame) is clearly seen in the flux-calibrated spectrum of J 2233-606 shown in Fig. 1 in Sealy et al. (1998) and its depth $\left(\tau_{\mathrm{c}}^{\mathrm{HI}} \sim 0.3\right)$ corresponds to $N(\mathrm{HI}) \sim 4 \times 10^{16} \mathrm{~cm}^{-2}$. A depression occurring in the flux-calibrated spectrum of HE 2347-4243 (Fig. 3 in Fechner et al. 2004) accounts for $N(\mathrm{H} \mathrm{I})$ from 3 to $7 \times$ $10^{16} \mathrm{~cm}^{-2}$. For HE 1341-1020, the corresponding wavelength range is beyond the observable frame, whereas for Q 0329-325 the flux-calibrated spectrum is not available. It should be emphasized that the flux-calibration is mandatory to detect this slow rolling continuum depression because otherwise it is disguised by instrumental effects. Further on, shallow troughs of hydrogen Ly- $\alpha$ and $\mathrm{O}$ VI absorptions extending over thousands of $\mathrm{km} \mathrm{s}^{-1}$ are present in spectra of HE 0141-1020 (Fig. 8, marked by horizontal lines), HE 2347-4243 (Fig. 12) and Q 0329-385 (Fig. 13). In spite of the very low optical depth of H I Ly- $\alpha\left(\tau_{\alpha}^{\mathrm{HI}} \sim 0.03\right)$, the corresponding portions can be clearly distinguished in the spectra due to the high signal-to-noise ratio and high spectral resolution which allows us to trace 'clear continuum windows' between absorption features over a wide spectral range. We also checked whether the detected troughs could be due to mismatch of echelle orders. The answer is no: in the spectral range considered the orders are small $\left(\sim 300 \mathrm{~km} \mathrm{~s}^{-1}\right)$, and a depression of the width of $\sim 1000 \mathrm{sm} \mathrm{s}^{-1}$ covers several subsequent orders.

The optical depth of an absorption line arising in a gas flow moving with a large velocity gradient is given by (Sobolev 1947, 1960; Castor et al. 1975):

$\tau=2.654 \times 10^{-15} f \lambda n /\left|\frac{\mathrm{d} v}{\mathrm{~d} s}\right|$,

where $f$ is the oscillator strength for absorption, $\lambda$ the central wavelength (in $\AA$ ), $n$ the number density (in $\mathrm{cm}^{-3}$ ) of atoms/ions in the ground state, $v$ the wind speed (in $\mathrm{km} \mathrm{s}^{-1}$ ), and $s$ the distance (in $\mathrm{cm}$ ) along the line of sight. The population of the upper excited level is ignored.

For H I Ly- $\alpha$ and O VI $\lambda 1031$ (as an example of a heavy element) the corresponding optical depths are

$$
\tau_{\alpha}^{\mathrm{HI}}=1.34 \times 10^{-12} n_{\mathrm{H}} \Upsilon_{\mathrm{HI}} /\left|\frac{\mathrm{d} v}{\mathrm{~d} s}\right|
$$

and

$\tau_{1031}^{\mathrm{OvI}}=3.63 \times 10^{-13} n_{\mathrm{H}} Z_{\mathrm{O}} \Upsilon_{\mathrm{OVI}} /\left|\frac{\mathrm{d} v}{\mathrm{~d} s}\right|$

where $Z_{\mathrm{O}}$ is the oxygen abundance and $\Upsilon_{\mathrm{HI}}, \Upsilon_{\mathrm{OVI}}$ are the ionization fractions of $\mathrm{H} \mathrm{I}$ and $\mathrm{O}$ VI, respectively. 

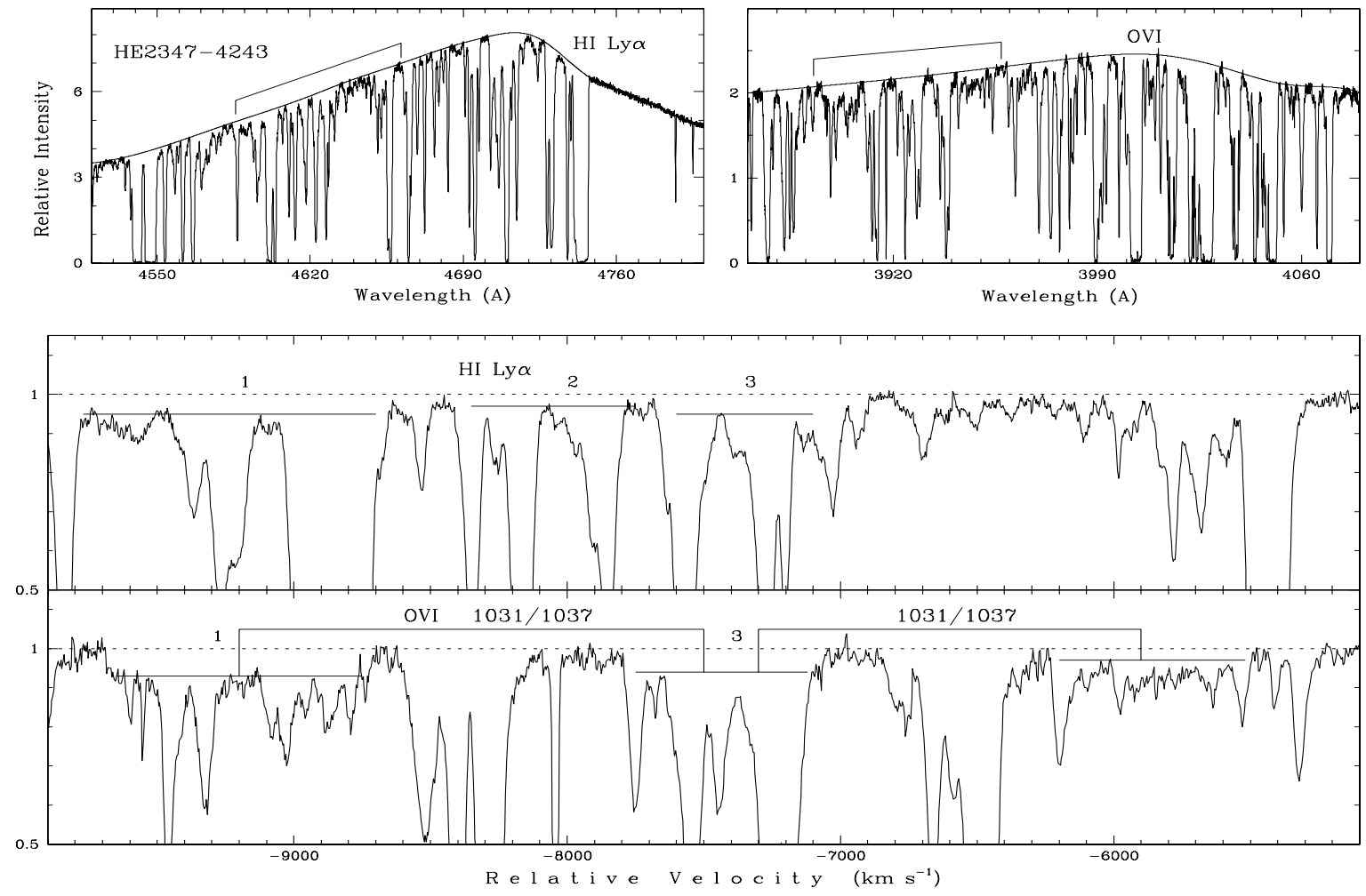

Fig. 12. Broad and shallow absorptions detected in the spectrum of HE 2347-4243. The zero radial velocity is fixed at $z=2.902$. For other details see caption to Fig. 8.

The viable estimation for the velocity acceleration $\mathrm{d} v / \mathrm{d} s$ at $v>1000 \mathrm{~km} \mathrm{~s}^{-1}$ is $10^{-7}-10^{-8} \mathrm{~km} \mathrm{~s}^{-1} \mathrm{~cm}^{-1}$ (e.g., Murray et al. 1995) with lower acceleration corresponding to higher velocities. Thus, to produce the optical depth of $\sim 0.03$ the number density of neutral hydrogen should be $n_{\mathrm{H}_{\mathrm{I}}} \sim(2-20) \times 10^{2} \mathrm{~cm}^{-3}$. For an ionization parameter $U \sim 1-5$ this gives the gas number density of $n_{\mathrm{H}}=10^{8}-10^{9} \mathrm{~cm}^{-3}$. Absorption in a gas with lower density will be indistinguishable from noise fluctuations in the quasar continuum windows whereas more dense parts of the disk wind will produce pronounced absorption which can be seen not only in HI, O VI, and Ne VIII but - due to lower $U-$ in such ions as C IV, NV or even Si IV as well. Large density fluctuations are expected in the disk wind due to flow stratification and due to the presence of various instabilities which give rise to shocks and dense shells as they propagate from the wind base outward and grow rapidly to the non-linear regime (Owocki et al. 1988; Proga et al. 2000). Thus, in general, the wind absorption is represented by a structure stretching over $1000 \mathrm{~s} \mathrm{~km} \mathrm{~s}^{-1}$ and consisting of a low-contrast continuum depression intermitted by parts without any absorption and by deeper absorption features caused by both density and velocity fluctuations ${ }^{3}$. In the quasar spectra considered here the broad and shallow absorption features can be detected at radial velocities up to $20000 \mathrm{~km} \mathrm{~s}^{-1}$ from the quasar's systemic redshifts which corresponds to $\Delta z \sim$ 0.2 at $2<z<3$. Thus, the superposition of absorption lines from the intervening Ly- $\alpha$ forest clouds is inevitable. Absorptions of $\mathrm{HI}$ and $\mathrm{O}$ VI in Figs. 8, 12, 13 show all elements of this picture ${ }^{4}$.

\footnotetext{
${ }^{3}$ Cf. Levshakov \& Kegel (1998), where a "line-like" structure caused by velocity fluctuations superposed on the general Hubble flow was calculated in the approximation of the constant gas density and neglecting the coupling of the velocity field with the radiation field.

${ }^{4}$ Similar complex absorption-line system consisting of shallow and broad $\left(F W H M \sim 700 \mathrm{~km} \mathrm{~s}^{-1}\right)$ and narrow $\left(F W H M<20 \mathrm{~km} \mathrm{~s}^{-1}\right)$
}

Another important question is to what extent the quasar's outcoming radiation is affected by the He II Lyman continuum absorption. It seems quite probable that outflow zones that produce BALs are opaque in He II continuum. X-ray observations of BAL quasars show that X-ray absorbing gas has $N(\mathrm{H})=$ $10^{22}-10^{24} \mathrm{~cm}^{-2}$ (Gallagher et al. 2002). The BAL systems are mostly identified by strong C IV absorption which supposes that the ionization parameter $U$ is less than 1 for a power law incident continuum (or that the incident continuum is soft at $E>4 \mathrm{Ryd}$ ). This gives a column density of neutral hydrogen of $N(\mathrm{H}) \sim$ $10^{17} \mathrm{~cm}^{-2}$ and of single ionized helium $N(\mathrm{He}$ II $) \sim$ few times of $10^{18} \mathrm{~cm}^{-2}$. Thus, the quasar spectrum transmitted through the BAL-producing region should have a pronounced break (more than 1 dex) at 4 Ryd. However, BAL quasars comprise only about $15 \%$ of the quasar population (Reichard et al. 2003) which means - if one considers the BAL effect as being due to the relative orientation of the disk and the line of sight - that BAL gas affects an insignificant part of the outcoming radiation.

As mentioned above, $N\left(\mathrm{He}\right.$ II) of $\sim 10^{18} \mathrm{~cm}^{-2}$ corresponds to $N(\mathrm{HI})$ of a few times $10^{16} \mathrm{~cm}^{-2}$. This amount of neutral hydrogen causes the continuum depression of $\sim 15-30 \%$ starting somewhere above $912 \AA$ in the rest-frame - the shielding gas can not only outflow but infall as well (Murray et al. 1995; Proga et al. 2000), shifting the depression to the red. For nonBAL QSOs, the presence of such a depression would indicate that there is enough He II to produce a noticeable intensity break at energies above 4 Ryd in the outcoming radiation. It seems natural to assume that the depth of this break depends on the angle to the rotational axis of the disk. A statistical study of the hydrogen Lyman continuum depression in the flux-calibrated

absorption lines displaced by $\simeq 14000 \mathrm{~km} \mathrm{~s}^{-1}$ blueward of the quasar systemic velocity was detected at $z=3.021$ towards CTQ 325 (Levshakov et al. 2004). 

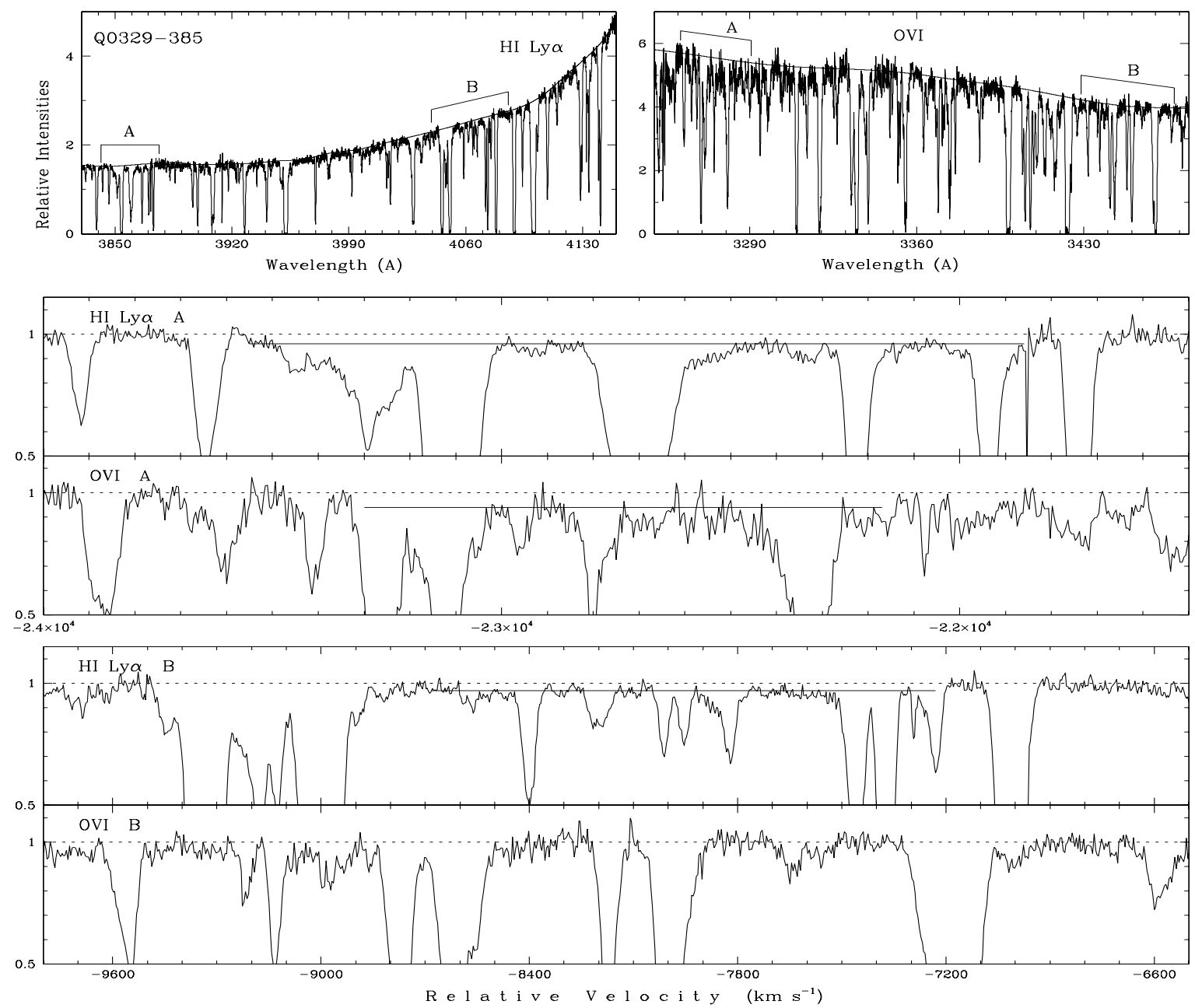

Fig. 13. Broad and shallow absorptions detected in the spectrum of Q 0329-385. The zero radial velocity is fixed at $z=2.435$. For other details see caption to Fig. 8.

high-resolution quasar spectra is expected to provide a comprehensive information about the covering factor of the He II opaque gas. This study is still to be done. However, there are several hints that this covering factor may indeed be significant. Continuum depression starting roughly at $\sim 930 \AA$ (rest-frame) is clearly seen in HST spectra of non-BAL quasars shown in Binette et al. (2005) who state that such quasars (named "class A" quasars) comprise about $60 \%$ of their sample (106 species). The estimates of the corresponding column densities of $\mathrm{HI}$ are not very certain due to the low quality of spectra, but nevertheless they vary between 2 and $6 \times 10^{16} \mathrm{~cm}^{-2}-$ well within the predicted boundaries. A good example shows a HST spectrum of HS $1103+6416$ presented in Fig. 1 in Köhler et al. (1999) - continuum depression starting at $\sim 950 \AA$ allows an accurate estimate of $N(\mathrm{HI}) \simeq 2.5 \times 10^{16} \mathrm{~cm}^{-2}$.

It is to note that the so-called "composite" spectra obtained by co-adding of many individual QSO spectra and shown in Fig. 5 in Telfer et al. (2002) and in Fig. 3 in Scott et al. (2004) are of no use in the present context since fine features present in an individual spectrum vanish or are smeared out in the co-added one. Moreover, before co-adding the individual spectra were 'statistically corrected' for the Ly- $\alpha$ forest absorption using the general distribution of the Ly- $\alpha$ clouds over $N(\mathrm{HI})$ and $z$. This operation is quite subjective because statistical properties of the Ly- $\alpha$ cloud distribution vary significantly from sightline to sightline. However, even in the composite spectra some feature are present which may be attributed to the quasar wind: a trough-like structure between 700 and $900 \AA$ in Fig. 5 (Telfer et al. 2002), and absorption features blueward of O VI and Ne VIII emission lines again in Fig. 5 (Telfer et al.) and in Fig. 3 in Scott et al. (2004). The former may be caused by the H I Lyman continuum absorption, and the latter by the resonance absorptions in the $\mathrm{O}$ VI and Ne VIII lines.

An additional support for possible He II opacity of quasar winds comes from X-ray observations which detect in $\sim 50 \%$ of quasars so-called "warm absorbers" - a broad absorption feature due to the blend of various ionization edges, among them O VII and O VIII (Piconcelli et al. 2005). There is evidence that these absorbers have $N(\mathrm{H}) \sim 10^{22}-10^{23} \mathrm{~cm}^{-2}$ and the ionization parameters $U \lesssim 5$ (Krongold et al. 2003; Schartel et al. 2005; Steenbrugge et al. 2005; Jimenez-Bailón et al. 2007). This leads again to $N(\mathrm{He}$ II $) \sim 10^{18} \mathrm{~cm}^{-2}$.

Thus, it seems quite likely that the outcoming QSO radiation in general is not a pure power law but has an intensity break at $E \geq 4$ Ryd due to the He II Lyman continuum absorption in the quasar disk wind. This can affect the rate of the He II reionization in the intergalactic clouds. Recent observations of the $\mathrm{HI} / \mathrm{He}$ II Ly $\alpha$ forest towards HE 2347-4342 (Shull et al. 2004; Zheng et al. 2004) and HS 1700+6416 (Fechner et al. 2006; Fechner \& Reimers 2007) revealed significant fluctuations of $\eta$ which suggest a variable softness parameter $S=J_{1 \text { Ryd }} / J_{4 \text { Ryd }}$ of the metagalactic radiation field. In this respect the revealed He II opacity 
of the quasar wind, which changes depending on angle to the rotational axis of the disk and differs also from quasar to quasar, can be responsible for the local fluctuations of $S$. For a pure power law ionizing spectrum, $F_{v} \propto v^{-\alpha}$, the photoionization rate of He II is $\Gamma_{1} \propto 1 /(\alpha+3)$. For a spectrum with a break at 4 Ryd, $\tau_{\mathrm{c}}^{\mathrm{He} \text { II }}>0$, the rate, $\Gamma_{2}$, becomes slower:

$\Gamma_{2} \propto \int_{1}^{\infty} \frac{\exp \left(-\tau_{\mathrm{c}}^{\mathrm{He} \text { II }} / x^{3}\right)}{x^{\alpha+4}} \mathrm{~d} x$, with $x=\frac{E}{4 \mathrm{Ryd}}$.

The ratio $\Gamma_{1} / \Gamma_{2}$ as a function of $\tau_{\mathrm{c}}^{\mathrm{He}}$ II is shown in Fig. 14 .

\subsection{Element abundances in quasar associated systems}

The properties of the NAL systems themselves are rather a byproduct of the present study. However, there are some interesting results obtained which are worth to be mentioned.

The metal content as well as the relative elemental abundances $(\mathrm{N} / \mathrm{C}, \alpha$-element/C, etc.) measured from the absorption systems are usually used to conclude about the enrichment mechanisms of the absorbing gas. Concerning the quasar-related absorption systems analyzed in the present work, the measured ratio $\mathrm{N} / \mathrm{C}$ shows that these mechanisms can be different for gas in the vicinity of the central engine and in the host galaxy. For instance, the systems at $z_{\text {abs }}=2.198(\mathrm{~J} 2233-606), z_{\text {abs }}=2.107$ (HE 1341-1020) and $z_{\mathrm{abs}}=2.352$ (Q 0329-385), all with systemic velocities of thousands of $\mathrm{km} \mathrm{s}^{-1}$ and, thus, parts of the outflows from the central region, reveal oversolar metallicity along with the relative overabundance of nitrogen as compared to solar values, $[\mathrm{N} / \mathrm{C}]>0$. On the other hand, the systems at $z_{\mathrm{abs}}=2.147(\mathrm{HE} 1341-1020)$ and $z_{\mathrm{abs}}=2.898(\mathrm{HE} 2347-4243)$, with the systemic velocities of $\sim 100-200 \mathrm{~km} \mathrm{~s}^{-1}$ and belonging probably to the gas in the host galaxy (cf. Nagao et al. 2006a), demonstrate similar oversolar metal content, but underabundant nitrogen, $[\mathrm{N} / \mathrm{C}]<0$. Overabundance of nitrogen, a common finding also in the analysis of quasar broad emission lines (e.g., Dietrich et al. 2003; Nagao et al. 2006b), is supposed to be a consequence of the rapid stellar evolution of quasar gas which leads to scaling of the N/O ratio with metallicity (Hamann \& Ferland 1999). However, different ratios [N/C] at similar metallicity measured in the circumnuclear gas and in the host galaxy indicates different enrichment mechanisms which may be due to variations in the initial mass function. Note that underabundance of nitrogen not complying with theoretical predictions of [N/C] and $[\mathrm{Fe} / \mathrm{C}]$ was measured in the associated absorption systems towards HE 0141-3932 (Reimers et al. 2005).

\section{Conclusions}

We have analyzed associated metal absorption systems identified in the UVES/VLT high resolution spectra $\left(F W H M \sim 6 \mathrm{~km} \mathrm{~s}^{-1}\right)$ of four quasars J 2233-606, HE 1341-1020, HE 2347-4243, and Q 0329-385 with the aim to restore the spectral shape of the underlying ionizing continuum in the EUV range $1<E<10$ Ryd. The systems were selected based on criteria that included the presence of lines of different ions and the possibility to estimate accurate covering factors and column densities. This ensured the reliability and robustness of the restored SEDs. All systems are physically related to the quasar/host galaxy and, thus, the SEDs in question are representative for the quasar outcoming radiation.

The results obtained can be summarized as follows.

1. The ionizing spectra responsible for the ionization structure in the analyzed systems reveal a sharp intensity depression

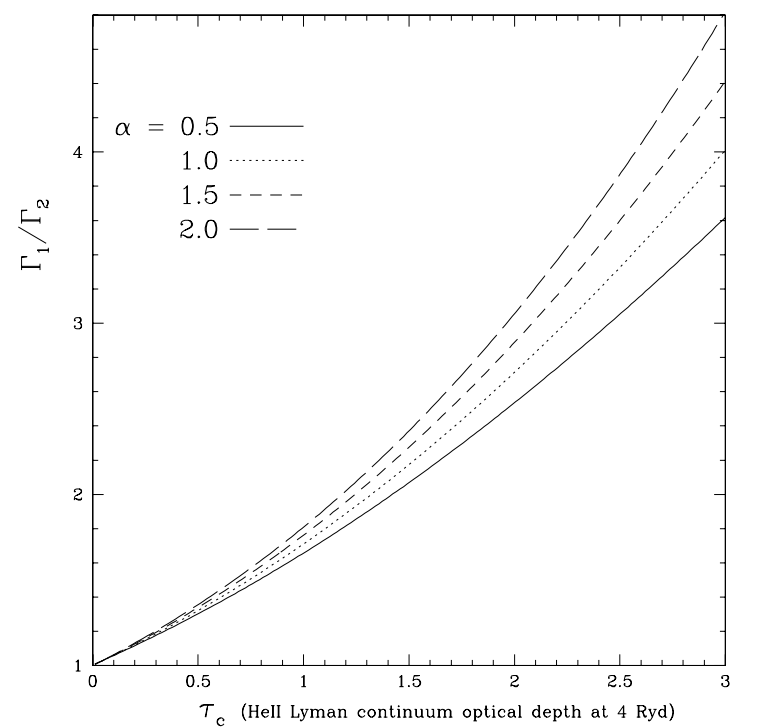

Fig. 14. The ratio of the photoionization rates $\Gamma_{1}$ (a pure power law spectrum $F_{v} \propto v^{-\alpha}$ ) to $\Gamma_{2}$ (a power law spectrum with a break at 4 Ryd) as a function of $\tau_{\mathrm{c}}^{\mathrm{He} I I}$.

at $E>4$ Ryd which is attributed to the absorption in the He II Lyman continuum. The required column density of He II is of the order of $10^{18} \mathrm{~cm}^{-2}$.

2. The most probable source of the He II opacity is the quasar accretion disk wind - a stratified flow comprising dense near-equatorial gas accelerated by combined action of radiation-pressure and magneto-centrifugal forces (seen as UV BAL absorption) and more highly ionized shielding gas that protects the inner wind regions from overionization by X-rays from the central source. None of the objects considered here belong to BAL-quasars which means that the He II opacity is due to the shielding gas. Assuming a pure power law SED at the wind base, we can estimate the column density of neutral hydrogen corresponding to $N($ He II $) \sim 10^{18} \mathrm{~cm}^{-2}: N(\mathrm{HI}) \sim$ a few times $10^{16} \mathrm{~cm}^{-2}$. This amount of neutral hydrogen can be seen in a quasar spectrum as a weak continuum depression starting at $\lambda 912 \AA$ (rest-frame) and sometimes also as broad (stretching over $1000 \mathrm{sm} \mathrm{s}^{-1}$ ) and shallow absorption in $\mathrm{H} \mathrm{I} \mathrm{Ly-} \alpha$. In the case of high metallicity (solar to oversolar) of the wind, the absorption features due to resonance lines of O VI in the FUV, $\mathrm{Ne}$ VI-Ne VIII in the EUV, and O VII-O VIII in the soft X-ray ranges are expected. In the UV spectra of quasars studied in the present paper, we do find broad and shallow H I Ly$\alpha$ and O VI $\lambda \lambda 1031,1037$ absorption features (Ne VI-Ne VIII beyond the available wavelength coverage) as well as continuum depression at $\lambda \sim 912 \AA$ in the flux-calibrated spectra of J 2233-606 and HE 2347-4243 (flux-calibrated spectra of other analyzed quasars are not available).

3. In order to estimate the fraction of quasar outcoming radiation affected by the He II Lyman continuum absorption, a systematic study of the predicted continuum depression in the flux-calibrated high-resolution QSO spectra at wavelengths shorter than $912 \AA$ (rest-frame) should be performed. This study is still to be done. However, observational data available now both in UV and X-ray regions suggest that at least $\sim 50 \%$ of the quasar radiation passes through the gas opaque in the He II Lyman continuum. This means that the outcoming power law ionizing continuum has a pronounced 
intensity break at $E>4$ Ryd with the depth of this break depending on the angle to the rotational axis of the accretion disk (the closer to the disk the deeper the break). This can influence the rate of the He II reionization in the intergalactic medium and partly explain the inhomogeneous (patchy) ionization structure of the intergalactic He II observed at $z \sim 3$.

4. Due to careful selection and detailed analysis of the absorption systems we obtained quite accurate estimates of the elemental abundances in the absorbing gas. It is shown that the relative abundance of nitrogen to carbon, $\mathrm{N} / \mathrm{C}$, differs for the circumnuclear and interstellar gas of the host galaxy: at similar oversolar metallicity the circumnuclear gas is overabundant in nitrogen, whereas the galactic gas is underabundant. This supposes different metal enrichment mechanisms which may be due to different IMF in the regions near the center and at the periphery of the quasar host galaxy.

Acknowledgements. We thank D. I. Nagirner, S. I. Grachev, and W. H. Kegel for useful discussions. I.I.A. and S.A.L. gratefully acknowledge the hospitality of the Shanghai Astronomical Observatory and Hamburger Sternwarte while visiting there. This research has been supported by the RFBR grant No. 06-02-16489, and by the Federal Agency for Science and Innovations grant NSh 9879.2006.2. J.L.H. is supported by NFC of China No. 10573028, and by 973 program with No. 2007CB815402.

\section{References}

Agafonova, I. I., Centurión, M., Levshakov, S. A., \& Molaro, P. 2005, A\&A, 441,9

Agafonova, I. I., Levshakov, S. A., Reimers, D., et al. 2007, A\&A, 461, 893 Asplund, M., Grevesse, N., \& Sauval, A. J. 2004, Nucl. Phys. A, 777, 1 Bahcall, J. N., \& Wolf, R. A. 1968, ApJ, 152, 701

Bergeron, J., Aracil, B., Petitjean, P., \& Pichon, C. 2002, A\&A, 396, L11

Binette, L., Magris, C. G., Krongold, Y., et al. 2005, ApJ, 631, 661

Brocksopp, C., Starling, R. L. C., Schady, P., et al. 2006, MNRAS, 366, 953

Castor, J. I., Abbott, D. C., \& Klein, R. I. 1975, ApJ, 195, 157

Costantini, E., Kaastra, J. S., Arav, N., et al. 2007, A\&A, 461, 121

Cristiani, S., \& D'Odorico, V. 2000, AJ, 120, 1648

Dietrich, M., Hamann, F., Shileds, J. C., et al. 2003, ApJ, 589, 722

D'Odorico, V., Cristiani, S., Donatella, R., Granato, G. L., \& Danese, L. 2004, MNRAS, 351, 976

Everett, J. E. 2005, ApJ, 631, 689

Fechner, C., \& Reimers, D. 2007, A\&A, 461, 847

Fechner, C., Baade, R., \& Reimers, D. 2004, A\&A, 418, 857

Fechner, C., Reimers, D., Kriss, G. A., et al. 2006, A\&A, 455, 91

Ferland, G. J., Korista, K. T., Verner, D. A., et al. 1998, PASP, 110, 761

Gabel, J. R., Arav, N., \& Kim, T.-S. 2006, ApJ, 646, 742

Gallagher, S. C., Brandt, W. N., Chartas, G., \& Garmire, G. P. 2002, ApJ, 567, 37

Haardt, F., \& Madau, P. 1996, ApJ, 461, 20

Hamann, F., \& Ferland, G. 1999, ARA\&A, 37, 487

Henry, R. B. C., Edmunds, M. G., \& Koppen, J. 2000, ApJ, 541, 660
Holweger, H. 2001, in Solar and Galactic Composition, ed. R. F. Wimmer-Schweingruber, AIP Conf. Proc., 598, 23

Jimenez-Bailón, E., Guainazzi, M., Matt, G., et al. 2007 [arXiv: 0710.1043]

Köhler, S., Reimers, D., Tytler, D., et al. 1999, A\&A, 342, 395

Levshakov, S. A., \& Kegel, W. H. 1998, MNRAS, 301, 323

Levshakov, S. A., Agafonova, I. I., \& Kegel, W. H. 2000, A\&A, 360, 833

Levshakov, S. A., Agafonova, I. I., Centurión, M., \& Molaro, P. 2003, A\&A, 397,851

Levshakov, S. A., D’Odorico, S., Agafonova, I. I., \& Dessauges-Zavadsky, M. 2004, A\&A, 413, 827

Mathews, W. G., \& Ferland, G. J. 1987, ApJ, 323, 456

Misawa, T., Charlton, J. C., Eracleous, M., et al. 2007, ApJS, 171, 1

Morris, S. L., Weymann, R. J., Foltz, C. B., et al. 1986, ApJ, 310, 40

Morton, D. C. 2003, ApJS, 149, 205

Murray, N., Chiang, J., Grossman, S. A., \& Voit, G. M. 1995, ApJ, 451, 498

Nava, A., Casebeer, D., Henry, R. B. C., \& Evremovic, D. 2007, ApJ, 645, 1076

Nagao, T., Maiolino, R., \& Marconi, A. 2006a, A\&A, 447, 863

Nagao, T., Maiolino, R., \& Marconi, A. 2006b, A\&A, 459, 85

Nicastro, F., Fiore, F., Perola, G. C., \& Elvis, M. 1999, ApJ, 512, 184

Osterbrock, D. E. 1974, Astrophysics of gaseous nebulae (San Francisco: W. H. Freeman and Co.)

Owocki, S. P., Castor, J. I., \& Rybicki, G. B. 1988, ApJ, 335, 914

Petitjean, P., \& Srianand, R. 1999, A\&A, 345, 73

Piconcelli, E., Jimenez-Bailón, E., Guainazzi, M., et al. 2005, A\&A, 432, 15

Proga, D. 2007, in The Central Engine of Active Galactic Nuclei, ed. L. C. Ho, \& J.-M. Wang, ASP Conf. Ser., 373, 267

Proga, D., Stone, J. M., \& Kallman, T. R. 2000, ApJ, 543, 686

Reichard, T. A., Richards, G. T., Hall, P. B., et al. 2003, AJ, 126, 2594

Reimers, D., Kohler, S., Wisotzki, L., et al. 1997, A\&A, 327, 890

Reimers, D., Köhler, S., Hagen, H.-J., \& Wisotzki, L. 1998, in Ultraviolet Astrophysics Beyond the IUE Final Archive, ed. W. Wamsteker, \& R. Gonzalez-Riestra (Noordwijk: ESA), 579

Reimers, D., Janknecht, E., Fechner, C., et al. 2005, A\&A, 435, 17

Reimers, D., Agafonova, I. I., Levshakov, S. A., et al. 2006, A\&A, 449, 9

Riess, A. G., Strolger, L.-G., Tonry, J., et al. 2004, ApJ, 607, 665

Schaye, J., Carswell, R. F., \& Kim, T.-S. 2007, MNRAS, 379, 1169

Sealey, K. M., Drinkwater, M. J., \& Webb, J. K. 1998, ApJ, 499, L135

Scott, J. E., Kriss, G. A., Brotherton, M., et al. 2004, ApJ, 615, 135

Schartel, N., Rodríguez-Pascual, P. M., Santos-Lleó, M., et al. 2005, A\&A, 433, 455

Smette, A., Heap, S. R., Williger, G. M., et al. 2002, ApJ, 564, 542

Shull, J. M., Tumlinson, J., Giroux, M. L., Kriss, G. A., \& Reimers, D. 2004, ApJ, 600, 570

Silva, A. I., \& Viegas, S. M. 2002, MNRAS, 329, 135

Sobolev, V. V. 1947, Moving Envelopes of Stars, Leningrad University Press (re-published by Harvard University Press in 1960)

Steenbrugge, K. C., Kaastra, J. S., Crenshaw, D. M., et al. 2005, A\&A, 434, 569

Steffen, A. T., Strateva, I., Brandt, W. N., et al. 2006, AJ, 131, 2826

Sutherland, R. S., \& Dopita, M. A. 1993, ApJS, 88, 253

Telfer, R. C., Zheng, W., Kriss, G. A., \& Davidsen, A. F. 2002, ApJ, 565, 773

Tripp, T. M., Lu, L., \& Savage, B. D. 1996, ApJS, 102, 239

Tripp, T. M., Jenkins, E. B., Williger, G. M., et al. 2002, ApJ, 575, 697

Verner, D. A., Barthel, P. D., \& Tytler, D. 1994, A\&AS, 108, 287

Zheng, W., Kriss, G. A., Telfer, R. C., Grimes, J. P., \& Davidsen, A. F. 1997, ApJ, 475, 469

Zheng, W., Kriss, G. A., Deharveng, J.-M., et al. 2004, ApJ, 605, 631 